OPEN ACCESS

Edited by: Xihui Shen,

Northwest A\&F University, China

Reviewed by:

Erik Petersen,

East Tennessee State University,

United States

Mofei Li,

Tianjin Normal University, China

Roshni R. Kharadi,

Michigan State University,

United States

*Correspondence:

Qin Liu

qinliu@ecust.edu.cn

Specialty section:

This article was submitted to

Bacteria and Host,

a section of the journal

Frontiers in Cellular and

Infection Microbiology

Received: 30 November 2021

Accepted: 13 January 2022

Published: 04 February 2022

Citation:

Wen Y, Wang Y, Chen S, Zhou X, Zhang $Y$, Yang $D$, Núñez G and Liu Q (2022) Dysregulation of Cytosolic c-di-GMP in Edwardsiella piscicida Promotes Cellular Non-Canonical Ferroptosis. Front. Cell. Infect. Microbiol. 12:825824. doi: 10.3389/fcimb.2022.825824

\section{Dysregulation of Cytosolic c-di-GMP in Edwardsiella piscicida Promotes Cellular Non-Canonical Ferroptosis}

\author{
Ying Wen ${ }^{1,2}$, Ying Wang ${ }^{1}$, Shouwen Chen ${ }^{1}$, Xiangshan Zhou ${ }^{1}$, Yuanxing Zhang ${ }^{3}$, \\ Dahai Yang ${ }^{1,4}$, Gabriel Núñez ${ }^{2}$ and Qin Liu ${ }^{1,4 *}$ \\ 1 State Key Laboratory of Bioreactor Engineering, East China University of Science and Technology, Shanghai, China, \\ 2 Department of Pathology and Comprehensive Cancer Center, University of Michigan, Ann Arbor, MI, United States, \\ ${ }^{3}$ Southern Marine Science and Engineering Guangdong Laboratory (Zhuhai), Zhuhai, China, ${ }^{4}$ Shanghai Engineering \\ Research Center of Maricultured Animal Vaccines, Shanghai, China
}

Programmed cell death plays an important role in modulating host immune defense and pathogen infection. Ferroptosis is a type of inflammatory cell death induced by intracellular iron-dependent accumulation of toxic lipid peroxides. Although ferroptosis has been associated with cancer and other sterile diseases, very little is known about the role of ferroptosis in modulating host-pathogen interactions. We show that accumulation of the secondary messenger bis- $\left(3^{\prime}, 5^{\prime}\right)$-cyclic dimeric GMP (c-di-GMP) in the pathogenic bacterium Edwardsiella piscicida (E. piscicida) triggers a non-canonical ferroptosis pathway in infected HeLa cells. Moreover, we observed that the dysregulation of c-diGMP in E. piscicida promotes iron accumulation, mitochondrial dysfunction, and production of reactive oxygen species, all of which that can be blocked by iron chelator. Importantly, unlike classical ferroptosis that is executed via excess lipid peroxidation, no lipid peroxidation was detected in the infected cells. Furthermore, lipoxygenases inhibitors and lipophilic antioxidants are not able to suppress morphological changes and cell death induced by E. piscicida mutant producing excess c-di-GMP, and this c-di-GMP dysregulation attenuates bacterial virulence in vivo. Collectively, our results reveal a novel non-canonical ferroptosis pathway mediated by bacterial c-di-GMP and provide evidence for a role of ferroptosis in the regulation of pathogen infection.

Keywords: Edwardsiella piscicida, c-di-GMP accumulation, non-canonical ferroptosis, bacterial virulence, pathogen infection

\section{INTRODUCTION}

Programmed cell death is comprised of non-lytic, immunologically silent forms of cellular demise such as apoptosis and lytic and pro-inflammatory forms of necrosis that include pyroptosis, necroptosis, and ferroptosis (Jorgensen et al., 2017). Ferroptosis is a more recently described modality of regulated necrosis, which is triggered by intracellular iron accumulation and excess peroxidation of polyunsaturated fatty acids through nonenzymatic reactions and lipoxygenase (LOX)-dependent enzymatic mechanisms (Dixon et al., 2012; Hassannia et al., 2019). Ferroptosis is 
regulated by glutathione peroxidase 4, a lipid repair enzyme capable of detoxifying hydroperoxides in complex lipids, and is induced by the inhibition or loss of glutathione peroxidase 4 activity (Dixon et al., 2012; Hassannia et al., 2019). A key feature of ferroptosis is that it can be blocked by iron chelators and lipophilic anti-oxidants (Dixon et al., 2012; Yang et al., 2014; Hassannia et al., 2019; Li et al., 2020). However, the specific molecular mechanisms that mediate ferroptosis remain poorly understood.

Increasing evidence has demonstrated the important dual function of programmed cell death in modulating host immune defense and pathogen infection. The death of an infected cell promotes the elimination of intracellular pathogen and the release of intracellular contents including cytokines and DAMPs, which further contributes to pathogen clearance and host immune defense. For instance, the intracellular host pattern recognition receptor NLRC4 can sense bacterial flagellins of various bacteria including Legionella pneumophila (Katagiri et al., 2012), Listeria Monocytogenes (Cervantes et al., 2008; Warren et al., 2008), Burkholderia thiailandensis (Miao et al., 2010), and Salmonella typhimurium (Yang et al., 2013) triggering caspase 1-mediated pyroptosis and secretion of the inflammatory cytokines IL-1 $\beta$ and IL-18 which enhances immune responses and limits bacterial invasion. Conversely, pathogens have evolved many strategies to manipulate cell death for successful infection. For example, Yersinia pestis prevents inflammasome activation by secreting YopK to interfere with pathogen recognition by NLRP3 and NLRC4, limiting host cell death and pathogen clearance (Dewoody et al., 2011). Thus, the question of whether and how cell death performs a physiologic function during infection is a key to understand pathogen-host interaction and bacterial pathogenesis, and develop therapeutic approaches to control infection. The role of apoptosis, pyroptosis and necroptosis in the regulation of host-pathogen interactions has been extensively studied (Labbe and Saleh, 2008; Ashida et al., 2011; Jorgensen et al., 2017; Imre, 2020) The role of ferroptosis in cancer and other sterile conditions such as neurodegeneration and acute kidney injury has been reported (Li et al., 2020). However, very little is known about the function of ferroptosis during pathogen infection.

The intracellular marine pathogen Edwardsiella piscicida (E. piscicida) infects a wide range of hosts from fish to birds, reptiles, and humans (Mohanty and Sahoo, 2007; Leung et al., 2012). E. piscicida causes a range of diseases, from ascites to immune organ dysfunction as well as persistent systemic infection in fish (Leung et al., 2019). Upon infection, host NLRC4 and NLRP3 can sense $E$. piscicida type III secretion system needle protein leading to the activation of caspase 1-mediated inflammasomes and triggering pyroptosis, which promotes host immune defense (Chen et al., 2017). Moreover, E. piscicida Trxlp promotes NLRC4 inflammasome activation in macrophages (Xu et al., 2018) while hemolysin provokes non-canonical inflammasome activation and pyroptosis by promoting intracellular sensing of LPS in mammals and fish (Chen et al., 2018; Wen et al., 2019). Conversely, E. piscicida has evolved diverse strategies to inhibit cell death and evade host immune recognition and killing. For instance, E. piscicida inhibits NLRP3 inflammasome activation via a type VI secretion system effector EvpP by regulating intracellular $\mathrm{Ca}^{2+}$ influx and JNK phosphorylation (Chen et al., 2017). Intracellular E. piscicida was also found to alter its arginine metabolism to facilitate cytosolic spermine accumulation and ultimately block NLRP3 inflammasome activation and pyroptosis (Jiang et al., 2021). Moreover, the type III secretion system effector EseJ of E. piscicida was reported to inhibit apoptosis by down regulating type 1 fimbriae during infection ( $\mathrm{He}$ et al., 2020). Collectively, these observations suggest that regulation of cell death is important during E. piscicida infection. However, the existence of additional cell death events during pathogen infection remains largely unknown.

Here we report that dysregulation of diguanylate cyclase (DGC) in E. piscicida induces host cell death with rupture of the cell membrane which is associated with attenuated virulence in zebrafish. We show that the activation of DGC results in elevated bis- $\left(3^{\prime}, 5^{\prime}\right)$-cyclic dimeric GMP (c-di-GMP) in $E$. piscicida triggering an iron-dependent, oxidative ferroptosis, which is independent of lipid oxidation. Our results indicate that tight regulation of intracellular c-di-GMP is critical for infection and suggest the existence of a non-canonical ferroptotic pathway independent of lipid peroxidation that can be triggered by pathogen infection.

\section{MATERIALS AND METHODS}

\section{Bacterial Strains and Growth Conditions}

The wild-type E. piscicida EIB202 (CCTCCM208068) has been described previously (Wang et al., 2009). E. piscicida 1906I was screened from the E. piscicida EIB202 transposon insertion mutant library (Yang et al., 2017) E. piscicida $\Delta 1906$ strain was constructed by unmarked depletion of gene ETAE_1906 in EIB202 strain while E. piscicida 1906I $\Delta 1905$ was constructed by depletion of gene ETAE_1905 in mutant 1906I. All the E. piscicida strains were inoculated at 1:100 and cultured statically for $12 \mathrm{~h}$ in tryptic soy broth (TSB) containing $16.7 \mu \mathrm{g} / \mathrm{ml}$ colistin at $30^{\circ} \mathrm{C}$. Escherichia coli DH5 $\alpha$, cc118 $\lambda$ pir, SM10 $\lambda$ pir, and Stbl 3 strains were used for the construction of plasmids and the conjugation of the pir-dependent suicide plasmid. E. coli strains were grown in Lysogeny broth or agar at $37^{\circ} \mathrm{C}$.

\section{Preparation of E. piscicida Mutants}

A previous in-frame gene mutation and deletion method based on allelic exchange was used to generate specific gene-deletion mutants of E. piscicida (Edwards et al., 1998). The upstream and downstream fragments of each indicated gene were fused by overlapping PCR and cloned into the $s a c B$ suicide vector $\mathrm{pDMK}$ and subsequently linearized with $B g l I I$ and SphI. Then the resulting plasmids were transformed into E. coli CC118 $\lambda$ pir. The correct plasmids were then transformed into E. coli SM10 $\lambda$ pir and conjugated into EIB202. The single-crossover mutants in which the plasmids have integrated into the chromosome by homologous recombination, were selected on tryptic soy agar plates containing kanamycin $(50 \mu \mathrm{g} / \mathrm{ml})$ or colistin $(16.7 \mu \mathrm{g} / \mathrm{ml})$. Then tryptic soy agar plates containing $10 \%$ sucrose 
were used to screen for the deletion mutants that completed allelic exchange. PCR amplification of the respective DNA loci and DNA sequencing of each PCR product were used to confirming the mutants. Primer pairs for generating upstream and downstream fragments of gene ETAE_1906 or ETAE_1905 are listed in Table S1.

\section{Generation of MlkI-Knockout Cell Lines}

Lentiviral CRISPR/Cas9 targeting guide RNA expressing vector (lentiCRISPRv2) was obtained from Addgene (\#52961). The $\mathrm{mlkl}$ knockout target sequence used was 5'-GCATATTATCACCCT TGGCC-3' (design by: http://zifit.partners.org). To generate gene knockout cell line, firstly lentiCRISPRv2 harboring the gene target sequence was constructed and transfected into 293T cells together with packing plasmids $\Delta 8.91$ and VSVG and with a ratio of 5:3:2. The supernatant of 293T cells which containing lentiviral particles was collected $48 \mathrm{~h}$ after transfection and then was used to infect HeLa cells for another $48 \mathrm{~h}$. Puromycinresistant cells were then diluted to 5 cells $/ \mathrm{mL}$ and seeded to $96-$ well plates, followed by a several-days expansion to establish a new clonal cell line. The clones with mlkl-deficiency were identified by immunoblotting with anti-MLKL antibody.

\section{Cell Culture and Infections}

Wild-type HeLa, 293T, and NCI-H226 cells were all obtained from ATCC. The mlkl-deficient HeLa cell lines were generated as above, and gsdmd-deficient HeLa cells were kindly provided by Dr. Feng Shao (National Institute of Biological Sciences, Beijing). HeLa and 293T cells were cultured in Dulbecco's Modified Eagle's medium (Gibco) while NCI-H226 cells were cultured in RPMI 1640 medium (Gibco). All medium were supplemented with $10 \%$ (vol/vol) fetal bovine serum (Gibco) and $1 \%$ penicillinstreptomycin (Sigma-Aldrich). All cells were grown at $37^{\circ} \mathrm{C}$ in a $5 \% \mathrm{CO}_{2}$ incubator.

For bacterial infection, cells were seeded and grown overnight to a density of $1 \times 10^{5}$ cells per well of 24 -well plates. Then the culture medium was replaced with phenol red-free Opti-MEM (Gibco) medium and cells were incubated with indicated $E$. piscicida strains at a multiplicity of infection (MOI) of 25. Infection was then initiated by centrifuging the plate at $600 \times g$ for $10 \mathrm{~min}$ and incubating at $35^{\circ} \mathrm{C}$ in an atmosphere containing $5 \% \mathrm{CO}_{2}$. The time point after centrifugation was recorded as the $0 \mathrm{~h}$ post infection. To induce secondary pyroptosis or ferroptosis, cells were treated with $20 \mu \mathrm{M}$ staurosporine (Sigma-Aldrich) or $20 \mu \mathrm{M}$ RSL3 (MCE), respectively.

For caspase inhibitor assays, z-VAD-fmk (50 $\mu \mathrm{M}$; MedChemExpress (MCE)), z-YVAD-fmk (50 $\mu \mathrm{M}$; SigmaAldrich), z-VDVAD-fmk (50 $\mu \mathrm{M}$; APExBIO), z-DEVD-fmk (50 $\mu \mathrm{M}$; APExBIO), z-WEHD-fmk $(20 \mu \mathrm{M}$; APExBIO), zVEID-fmk (50 $\mu \mathrm{M}$; APExBIO), z-IETD-fmk (50 $\mu \mathrm{M}$; APExBIO), or $z$-LEHD-fmk (50 $\mu \mathrm{M}$; APExBIO) were incubated, respectively, with cells at $37^{\circ} \mathrm{C}$ for $1 \mathrm{~h}$ before bacterial addition. For other pharmacological inhibitors assays, cells were pretreated with cytochalasin D ( $2 \mu \mathrm{g} / \mathrm{ml}$; Invitrogen), azathioprine (50 $\mu \mathrm{g} / \mathrm{ml}$; Sigma-Aldrich), sulfathiazole $(100 \mu \mathrm{g} /$ ml; Sigma-Aldrich), Necrostatin-1 (Nec-1, $50 \mu \mathrm{M}$; MCE), GSK'872 (3 $\mu \mathrm{M}$; MCE), necrosulfonamide (NSA, $20 \mu \mathrm{M}$; MCE), deferoxamine mesylate (DFM, $50 \mu \mathrm{M}$; MCE),
PD146176 (5 $\mu \mathrm{M}$; Sigma-Aldrich), zileuton (5 $\mu \mathrm{M}$; MCE), ML351 (10 $\mu \mathrm{M}$; Cayman), ferrostatin-1 (Fer-1, $20 \mu \mathrm{M}$; MCE), or liprostatin-1 (Lipro-1, $20 \mu \mathrm{M}$; MCE), respectively, for $30 \mathrm{~min}$ before being exposed to bacteria or other chemicals.

\section{Cell Death Assay}

As described previously, a qualitative method based on cellular morphological observation and a quantitative method detecting the release cytosolic contents were used to examine cell death, respectively (Wen et al., 2019). Cells were treated as described previously in 24-well plates and the dynamic morphological changes of cells were captured using an Olympus IX71 fluorescent microscope at room temperature. Propidium iodide (PI, $5 \mathrm{ng} / \mathrm{ml}$ ) was added to the medium as an indicator of cell membrane integrity. Quantitative cytotoxicity was detected by measuring lactate dehydrogenase $(\mathrm{LDH})$ release of infected cells. The culture medium was collected at the indicated time points after bacterial infection and centrifuged at $600 \times g$ for $5 \mathrm{~min}$. Then $50 \mathrm{ul}$ clear supernatants were transferred to per well of 96-well plate (flat bottom) for subsequent LDH detection with a CytoTox 96 assay kit (Promega) according to the manual. Each sample was examined in triplicate and the absorbance at $490 \mathrm{~nm}$ was measured using a microplate reader (Dynex Technologies). The absorbance of supernatant from uninfected/untreated cells was used for background subtraction. Cytotoxicity was normalized to lysis buffer treatment (100\% of control). The percentage of cell death was calculated as follows: Percentage $(\%)$ of cell death $=($ A490 nm of samples - A490 nm of uninfected cells)/(A490 nm of lysis buffertreated cells - A490 nm of uninfected cells $) \times 100$.

\section{Zebrafish Infection}

The protocol for all the experiments on zebrafish here was approved by the Institutional Animal Care and Use Committee of East China University of Science and Technology (No. 2006272). Single colony of indicated E. piscicida strains was inoculated into TSB medium containing $16.7 \mu \mathrm{g} / \mathrm{ml}$ colistin and cultured overnight at $30^{\circ} \mathrm{C}$ in a shaking incubator with speed of $200 \mathrm{rpm}$, then inoculated at 1:100 into fresh pure TSB culturing statically at $30^{\circ} \mathrm{C}$ to an $\mathrm{OD}_{600}$ of 0.8 . Bacteria was pelleted by centrifugation $(4500 \times \mathrm{g})$ at $4^{\circ} \mathrm{C}$ for $10 \mathrm{~min}$ and washed three times with cold PBS, then suspended in PBS to $5000 \mathrm{CFU} / \mathrm{ml}$. Zebrafish (6 months old) were randomly selected and intramuscularly injected with $10 \mu \mathrm{l}$ bacterial suspension (50 CFU per fish) or PBS alone. Subsequently, the fish were transferred to clear tanks with a density of no more than 35 fish per tank and incubated at room temperature. The mortality was then recorded at the different time points. For bacterial colonization determination, five surviving zebrafish were pooled as a sample and dissected at the indicated post infection time points. Liver, spleen, kidneys, and intestines were collected and homogenized for CFU counts.

\section{Quantitative RT-qPCR}

For RT-qPCR, total bacterial RNA was isolated by using an RNA isolation kit (Tiangen) according to the instruction manual and concentration of extracted RNA was measured by a NanoDrop spectrophotometer (Thermo Scientific). Then FastKing One Step 
RT-PCR Kit (Tiangen) was used for RNA reverse transcription and cDNA synthesis. The kit SuperReal Pre-Mix Plus (SYBR Green; Tiangen) was used for quantitative real-time PCR, which was performed on a FTC-200 detector (Funglyn Biotech). All the procedures followed the instruction manuals. Gene expression was evaluated for three biological replicates, and the data for each sample were calculated relative to the expression level of the $16 \mathrm{~S}$ gene by using the $2^{-\triangle \mathrm{CCT}}$ method. Primers used in this study are listed in Table S2.

\section{Bacterial c-di-GMP Detection}

To quantitatively measure c-di-GMP level in E. piscicida strains, Cayman's cyclic di-GMP ELISA kit was used. All the indicated strains were cultured as described above. Bacteria was cultured statically for $12-16 \mathrm{~h}$ and then pelleted by centrifugation $(8000 \times$ $g$, $5 \mathrm{~min}$ ) at $4^{\circ} \mathrm{C}$, followed by triple wash with cold PBS to eliminate any organic components from culture medium. $8 \times 10^{9}$ bacterium were collected at final as for each strain and $500 \mu \mathrm{lB}-$ PER reagent (Thermo Fisher) was added to and mixed sufficiently with bacteria by pipetting. Incubating 15 mins at room temperature to lyse the bacteria, then clear supernatant of lysate was collected by centrifugation $(15000 \times \mathrm{g})$ at $4^{\circ} \mathrm{C}$ for $10 \mathrm{~min}$ and kept on ice for following steps. Protein in the lysate supernatants was determinized by Pierce BCA protein assay (Thermo Scientific) and c-di-GMP was quantitatively measured with cyclic-di-GMP ELISA kit according to the manufacturer's manuals. Bacterial c-di-GMP level was normalized to total protein amount of lysate supernatant.

\section{Detection of Bacterial Biofilm Formation and Exopolysaccharides (EPS) Production}

To measure bacterial biofilm formation, the indicated E. piscicida strains were cultured overnight in TSB containing colistin at $30^{\circ} \mathrm{C}$ in a shaking incubator with speed of $200 \mathrm{rpm}$. Then $10 \mu \mathrm{l}$ bacterial suspension in PBS with a concentration of $1 \times 10^{9} \mathrm{CFU} /$ $\mathrm{ml}$ was added into $200 \mu \mathrm{l}$ TSB in each well of sterile 96-well polystyrene plates. There were 6 replicates for each strain. Bacteria was cultured at $30^{\circ} \mathrm{C}$ for $24 \mathrm{~h}$. After that the culture fluid with planktonic bacteria was aspirated, and the wells were washed 3 times with PBS. The formed biofilm was fixed with 200 $\mu \mathrm{l}$ methanol for $15 \mathrm{~min}$ and stained by adding $200 \mu \mathrm{l}$ of $0.1 \%$ crystal violet water solution into each well for $30 \mathrm{~min}$ at room temperature. Unbounded dye was removed by triple washing with PBS. To extract the dye bound with the adsorbed biofilm, $200 \mu \mathrm{l} 95 \%$ ethanol was added to wells for $30 \mathrm{~min}$ and then absorbance at $590 \mathrm{~nm}$ of mixture was measured by a plate reader. A mixture of sterile PBS $(10 \mu \mathrm{l})$ and TSB $(200 \mu \mathrm{l})$ was used as the negative control for which the sequence of operations was same as for experimental wells, and the absorbance at $590 \mathrm{~nm}$ was used as a background subtraction. The relative biofilm formation ability was the ratio of the absorbance at $590 \mathrm{~nm}$ of indicated strains to that of wild-type strain EIB202.

The bacterial EPS production was quantified by Congo red binding assay. Overnight-cultured E. piscicida was collected and suspended with PBS with a concentration of $1 \times 10^{9} \mathrm{CFU} / \mathrm{ml}$ and $2 \mathrm{ml}$ bacterial broth were pelleted by centrifugation $(4500 \times g$, $10 \mathrm{~min}$ ) at $4^{\circ} \mathrm{C}$, followed by suspending and incubating with $1 \mathrm{ml}$
M9 medium containing $40 \mu \mathrm{g} / \mathrm{ml}$ Congo red at $30^{\circ} \mathrm{C}$ in a shaking incubator with speed of $180 \mathrm{rpm}$ for $3 \mathrm{~h}$. Then, supernatant was separated with bacteria by centrifuging at $8000 \times g$ for $5 \mathrm{~min}$. Aliquots of clear supernatant were transferred into a 96-well plate and the absorbance at $490 \mathrm{~nm}$ was measured. The absorbance of M9 medium alone was used as the background and should be subtracted. The absorbance of M9 medium containing $40 \mu \mathrm{g} / \mathrm{ml}$ Congo red was used as $100 \%$ of control. The bacterial EPS production positively correlated with Congo red adsorbed by bacteria which was calculated as following formula: Percentage of Congo red adsorption by bacteria $=(1$ $A_{\text {samples }}\left(A_{\text {control }}\right) \times 100 \%$.

\section{Assessment of Bacterial Adhesion and Internalization}

HeLa cells were seeded into 24 -well plate with density of $1 \times 10^{5}$ cells per well and infected with indicated E. piscicida strains at a MOI of 100 for 1 h. Followed by that, cells were washed with cold PBS for 3 times to remove the unattached bacteria. To assess bacteria adhesion, cells were then lysed with $1 \%$ Triton X-100 and the cell lysate containing attached bacteria was serially diluted and CFU was counted. The ability of bacterial adhesion was calculated as the ratio of CFU per cell. To assess bacterial internalization, cells were incubated with Opti-MEM containing with $800 \mu \mathrm{g} / \mathrm{ml}$ streptomycin for $0.5 \mathrm{~h}$ to kill extracellular bacteria and then washed with cold PBS for another 3 times followed by lysis with $1 \%$ Triton X-100. The cell lysate containing internalized bacteria was serially diluted and CFU was counted. The ability of bacterial internalization was calculated as the ratio of CFU per cell.

\section{Caspase Activity Assays}

The activity of caspases was assayed according to the substrate manufacturer's instructions. Briefly, HeLa cells were infected with $E$. piscicida EIB202 or $1906 \mathrm{I}$ for $3.5 \mathrm{~h}$ and collected by trypsindigesting and centrifuging $\left(600 \times \mathrm{g}, 5 \mathrm{~min}, 4^{\circ} \mathrm{C}\right)$, then lysed with lysis buffer provided in the caspase activity assay kit. Subsequently, chromogenic substrates (Beyotime Biotechnology) of human caspase-1 (Ac-YVAD-pNA), caspase-2 (Ac-VDQQDpNA), caspase-3/7 (Ac-DEVD-pNA), caspase-4 (Ac-LEVD-pNA), caspase-6 (Ac-VEID-pNA), caspase-8 (Ac-IETD-pNA), and caspase-9 (Ac-LEHD-pNA) were incubated with cell lysates at a final concentration of $200 \mu \mathrm{M}$, while a fluorescent substrate AcWEHD-AFC (BioVision) was used to detect the activity of caspase5 in lysate, which has an excitation at $400 \mathrm{~nm}$ and emission at 505 $\mathrm{nm}$. Caspase activity was examined by measuring changes in absorbance at $405 \mathrm{~nm}$ or fluorescence at $505 \mathrm{~nm}$ at 5-min intervals caused by the presence of free pNA hydrolyzed from the substrates. Uninfected HeLa cells was taken as a negative control and background subtraction. The relative caspase activity was the absorbance or fluorescence of cells infected with 1906I relative to the absorbance or fluorescence of EIB202 samples.

\section{Antibodies}

All antibodies were used for Western blotting. Rabbit anti-MLKL monoclonal antibody (1:1000; Abcam) was used to verify clones with MLKL deficiency while rabbit anti-GSDME-N-terminal 
monoclonal antibody (1:1000; Abcam) was used for detecting cleavage of GSDME. Mouse anti- $\beta$-actin monoclonal antibody (1:1000; Cell Signaling Technology) was used to indicate the loaded protein amount.

\section{Determination of Intracellular Labile Iron}

Fluorescent probes Calcien-AM (CA-AM, Invitrogen) and Phen green SK (PGSK, Invitrogen), which bind iron rapidly, stoichiometrically, reversibly while performing fluorescencequenched complexes (Epsztejn et al., 1997; Petrat et al., 1999), were used to quantitively measure cytosolic labile iron. Briefly, $1 \times 10^{5} \mathrm{HeLa}$ cells were seeded into 24 -well plate for overnight culture and incubated with DFM-added Opti-MEM or Opti$\mathrm{MEM}$ alone at $37^{\circ} \mathrm{C}$ for $30 \mathrm{~min}$ before infection of E. piscicida EIB202 or 1906I, or treatment of $200 \mu \mathrm{M}$ ferric ammonium citrate (FeAC). Control cells were only incubated with OptiMEM. At $3 \mathrm{~h}$ post incubation of bacteria or FeAC, cells were rinsed once with $\mathrm{PBS}$ and then incubated with Opti-MEM containing $250 \mathrm{nM}$ CA-AM or $20 \mu \mathrm{M}$ PGSK at $37^{\circ} \mathrm{C}$ for 20 min, respectively. The CA-AM and PGSK are nonfluorescent lipophilic, esterified precursors and rapidly permeate the plasma membrane. Once inside the cell, they are hydrolyzed to be fluorescent iron chelator. The fluorescence is then quenched immediately by intracellular iron. Excess CA-AM or PGSK that did not penetrate the cells was removed by triple wash with PBS and $500 \mu \mathrm{l}$ Opti-MEM was added to each well. Subsequently, CA-AM or PGSK fluorescence intensity of cells were measured with a Multiscan Spectrum microplate reader (Synergy H1, BioTek) at an excitation wavelength of $485 \mathrm{~nm}$ and an emission wavelength of $530 \mathrm{~nm}$. The quenched fluorescence intensity compared to untreated control cells reflected the cytosolic labile iron accumulated in cells after bacterial infection or FeAC treatment.

To measure labile iron in mitochondrial, cells were incubated with mitochondria-targeted fluorescence probe Mito-FerroGreen (Dojindo) in accordance with the manufacturer's instructions. Briefly, after appropriate treatments with DFM or infection with bacteria, HeLa cells were washed three times with HBSS at $3.5 \mathrm{~h}$ post infection and incubated with $5 \mu \mathrm{M}$ Mito-FerroGreen for $30 \mathrm{~min}$ at $37^{\circ} \mathrm{C}$ in a $5 \% \mathrm{CO}_{2}$ incubator. Followed by discarding the supernatant, HeLa cells were washed three times with HBSS and then dissociated by adding enzyme-free cell dissociation buffer (Gibco) and collected for flow cytometry analysis. Sample data was collected utilizing a BD FACSCelesta flow cytometer. 10000 events were collected for each sample after gating out debris and fluorescence of cells were measured using F-1 FITC channel. Data files were analyzed using FlowJo V10. Fluorescence intensity represented the labile iron in mitochondrial.

\section{Mitochondrial Potential Assay (JC-1)}

A fluorescent lipophilic carbocyanine dye JC-1 (MCE) was used to indicate changes of mitochondrial membrane potential $(\Delta \Psi \mathrm{m})$. JC-1 forms complexes known as J-aggregates at high $\Delta \Psi \mathrm{m}$ which emits an orange-red fluorescence $(\mathrm{Ex} / \mathrm{Em}=585 \mathrm{~nm} /$ $590 \mathrm{~nm})$. While in cells with low $\Delta \Psi \mathrm{m}$, JC-1 remains in the monomeric form emitting a green fluorescence $(\mathrm{Ex} / \mathrm{Em}=510 \mathrm{~nm} /$ $527 \mathrm{~nm})$. Briefly, $1 \times 10^{5} \mathrm{HeLa}$ cells were seeded into 24 -well plate and cultured overnight. Followed by appropriate treatments with DFM or infection with indicated E. piscicida strains, HeLa cells were washed three times with PBS at $3.5 \mathrm{~h}$ post infection and then incubated with Opti-MEM containing $2 \mu \mathrm{M} \mathrm{JC}-1$ at $37^{\circ} \mathrm{C}$ for $20 \mathrm{~min}$ in a $5 \% \mathrm{CO}_{2}$ incubator. After JC-1 staining, cells were washed three times with PBS and $500 \mu \mathrm{l}$ Opti-MEM was added to the wells. Fluorescence of JC-1 aggregates and monomers were observed and images were directly photographed by Olympus IX71 fluorescent microscope under FITC and TRITC filter.

\section{Cellular ATP Detection}

ATP level in cells with or without infection was quantitatively detected by using a luminescent ATP-based cell viability assay kit (Promega) according to the slightly modified instruction manual. Briefly, $1 \times 10^{5}$ HeLa cells were seeded into 24 -well plate and cultured overnight. Cells were pretreated with DFM or incubated with Opti-MEM alone at $37^{\circ} \mathrm{C}$ for $30 \mathrm{~min}$ and then infected with E. piscicida EIB202 or 1906I at a MOI of 25 for 3.5 h. Partial cell culture medium was removed leaving $200 \mu \mathrm{l}$ medium in the well. Then an equivalent volume of CellTirter-Glo 2.0 Reagent from Promega kit was added into each well and incubated for $10 \mathrm{~min}$ on an orbital shaker to induce cell lysis and reaction at room temperature. Subsequently, $100 \mu \mathrm{l}$ reaction buffer was transferred into an opaque-walled 96-well plate and luminescence was recorded which is proportional to the amount of ATP present in cells. The luminescence of mixture of CellTirter-Glo 2.0 Reagent with pure Opti-MEM was a background and should be subtracted when calculating luminescence of other samples.

\section{Mitochondrial and Cytosolic Reactive Oxygen Species (ROS) Measurement}

A fluorescent mitochondrial-targeted probe MitosOX (Invitrogen), oxidation of which by superoxide produces red fluorescence, was used to detect mitochondrial ROS production according to the instruction manual. Briefly, Cells were pretreated with DFM or incubated with pure Opti-MEM at $37^{\circ} \mathrm{C}$ for $30 \mathrm{~min}$ and then infected with E. piscicida EIB202 or $1906 \mathrm{I}$ at a MOI of 25 for $3.5 \mathrm{~h}$. After that, HeLa cells were washed once with HBSS and incubated with $5 \mu \mathrm{M}$ MitoSOX for $10 \mathrm{~min}$ at $37^{\circ} \mathrm{C}$ in a $5 \% \mathrm{CO}_{2}$ incubator. Subsequently, cells were washed gently with warm HBSS for three times and then dissociated by adding enzyme-free cell dissociation buffer (Gibco) and collected for flow cytometry analysis. Sample data were collected utilizing a BD FACSCelesta flow cytometer. 10000 events were collected for each sample after gating out debris and fluorescence of cells were measured using F-2 PE channel. Data files were analyzed using FlowJo V10. Fluorescence intensity represented mitochondrial oxidative stress.

DCFH-DA fluorescence probe (Beyotime) was used to assess cytosolic ROS. Once DCFH-DA permeates the plasma membrane, it is then deacetylated by cellular esterases to a non-fluorescent compound, which is later oxidized by ROS into highly green-fluorescent DCF. Based on this principle, cells with stronger oxidative stress exhibit higher value of green fluorescence. For analysis, cells were washed once with PBS after 3.5 hours' infection with E. piscicida strains and incubated with Opti-MEM containing $10 \mu \mathrm{M}$ DCFH-DA at $37^{\circ} \mathrm{C}$ for $20 \mathrm{~min}$ in a 
$5 \% \mathrm{CO}_{2}$ incubator. Subsequently, excess DCFH-DA outside the cells was removed by washing cells three times with Opti-MEM. Cell fluorescence was observed and images were captured with Olympus IX71 fluorescent microscope with laser at excitation wavelength $488 \mathrm{~nm}$ (FITC filter).

\section{Lipid Peroxidation Detection}

A lipid-soluble fluorescent indicator C11-BODIPY ${ }^{581 / 591}$ (Invitrogen) was used to detect lipid peroxidation. Non-oxidized C11-BODIPY ${ }^{581 / 591}$ can be excited at wavelength of $581 \mathrm{~nm}$ and emits red fluorescence $(\mathrm{Ex} / \mathrm{Em}=581 \mathrm{~nm} / 591 \mathrm{~nm})$. Upon oxidation, its excitation maximum shifts from 581 to $500 \mathrm{~nm}$ and the emission maximum shifts from $591 \mathrm{~nm}$ to $510 \mathrm{~nm}$, emitting green fluorescence. Briefly, $1 \times 10^{5} \mathrm{HeLa}$ cells were seeded into 24 -well plate and cultured overnight. Followed by appropriate treatments with RSL3 or infection with indicated E. piscicida strains, HeLa cells were washed three times with PBS at $3.5 \mathrm{~h}$ post infection or $20 \mu \mathrm{M}$ RSL3 treatment and then incubated with Opti-MEM containing 2 $\mu \mathrm{M} \mathrm{C} 11-\mathrm{BODIPY}{ }^{581 / 591}$ at $37^{\circ} \mathrm{C}$ for $30 \mathrm{~min}$ in a $5 \% \mathrm{CO}_{2}$ incubator. After staining, cells were washed three times with PBS and $500 \mu \mathrm{l}$ Opti-MEM was added to the wells. Fluorescence of non-oxidized and oxidized C11-BODIPY ${ }^{581 / 591}$ were observed and images were photographed by Olympus IX71 fluorescent microscope with lasers under FITC and TRITC filter.

\section{Statistical Analysis}

Data were presented as the mean \pm standard deviation (SD) of triplicate sample per experimental condition unless noted otherwise. Representative results were shown in the figures. Statistical analyses were performed using two-way ANOVA, one-way ANOVA, multiple $t$ test, and Gehan-BreslowWilcoxon test of GraphPad Prism Program (Graphpad Software). Error bars represent the standard deviation. Significant statistical differences were indicated by asterisks: ${ }^{\star} P<0.05 ;{ }^{\star \star} P<0.01 ;{ }^{\star \star \star} P<0.001$.

\section{RESULTS}

\section{A Transposon Insertion in E. piscicida Promotes Lytic Cell Death in HeLa Cells and Reduces Pathogen Virulence in Zebrafish}

To explore the physiologic function of cell death and the strategies used by pathogens to regulate host cell death during infection, we previously utilized a transposon insertion mutant library of E. piscicida to identify mutants that induce membraneruptured cell death based on cell morphology changes and LDH release (Chen et al., 2018; Wen et al., 2019). A novel E. piscicida mutant which was named 1906I was found to trigger cell death in HeLa cells upon infection. At the early period of infection (at $2.5 \mathrm{~h}$ post infection), HeLa cells infected with mutant 1906I began to exhibit some morphological features of apoptosis, such as membrane blebbing and formation of balloon-like protrusions (Figure 1A). Subsequently, 1906I-infected cells showed swelling and rounding, and began to show increased membrane permeability and loss of plasma membrane integrity by $3.5 \mathrm{~h}$ post infection. Cells exhibited membrane ruptured as the cytoplasm appeared red after incubation with the membraneimpermeable PI (Figure 1A). By contrast, the morphology of HeLa cells infected with wild-type E. piscicida EIB202 was similar to that of untreated cells (Figure 1A). Consistent with morphological changes shown with PI-positive staining, increased $\mathrm{LDH}$ release was detected in 1906I-infected HeLa cells during the late period of infection (at $5 \mathrm{~h}$ post infection) compared to that observed in EIB202-infected cells (Figure 1B). These results indicate that the transposon gene insertion in the $E$. piscicida 1906I mutant promotes robust cell death associated with ruptured membrane.

We next determined the function of the 1906I mutant after infection in vivo. To assess this question, we infected adult zebrafish with wild-type EIB202 or 1906I by intramuscularly injecting the fish with $50 \mathrm{CFU}$. We found that all zebrafish succumbed after infection with EIB202 whereas 1906I infected fish exhibited remarkably reduced mortality (Figure 1C). Consistent with reduced mortality, fish infected with 1906I mutant showed lower bacterial loads in the liver, spleen, kidney, and intestine on days 2 and 3 after infection than animals infected with wild-type EIB202 (Figures 1D-G). These results suggest that gene disruption by transposon insertion in the 1906 I mutant limits E. piscicida colonization in zebrafish.

\section{Upregulation of Cytosolic c-di-GMP in E. piscicida Induces Robust Membrane Rupture-Mediated Cell Death}

E. piscicida is an intracellular pathogen, but the 1906I-induced cell death was found to be independent of intracellular bacteria since 1906I showed lower internalization ability and inhibitor of endocytosis displayed no suppressive effect on cell death (Figure S1). To further investigate the mechanism by which 1906I induces lytic cell death, we analyzed the transposon insertion site within 1906I and found that the transposon was inserted in an operon containing the gene ETAE_1905 that encodes DGC (Figure 2A). DGC synthesizes the second messenger c-di-GMP from two GTP molecules which facilitates the biosynthesis of bacterial adhesins and EPS as well as biofilm formation by increasing c-di-GMP levels (Hengge, 2009). Bioinformatic analysis revealed the gene ETAE_1906 inactivated by transposon insertion was located upstream of $D g c$, the gene encoding DGC (ETAE_1905) (Figure 2A). Transcriptional analysis of genes adjacent to insertion site indicated significant upregulation of ETAE_1905 in 1906I compared with the wild-type EIB202 (Figure S2). In agreement with upregulated transcription levels of ETAE_1905, 1906I produced higher c-di-GMP levels than EIB202 (Figure 2B). Consistent with increased levels of c-di-GMP, the 1906I mutant showed enhanced biofilm formation (Figure 2C), EPS production (Figure 2D) and adhesion to HeLa cells (Figure 2E). In contrast, deletion of ETAE_1906 in EIB202 decreased slightly the c-di-GMP levels, but did not affect biofilm formation, exopolysaccharide production or adhesion to HeLa cells compared with EIB202 (Figures 2B-E). Importantly, deletion of ETAE_1905 in the 1906I mutant greatly impaired c-di-GMP levels and the increased 
A
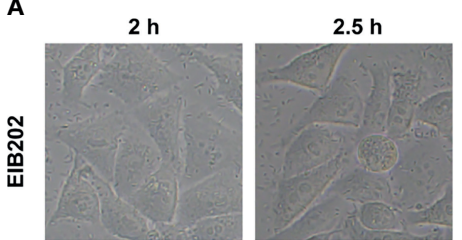

$3.5 \mathrm{~h}$
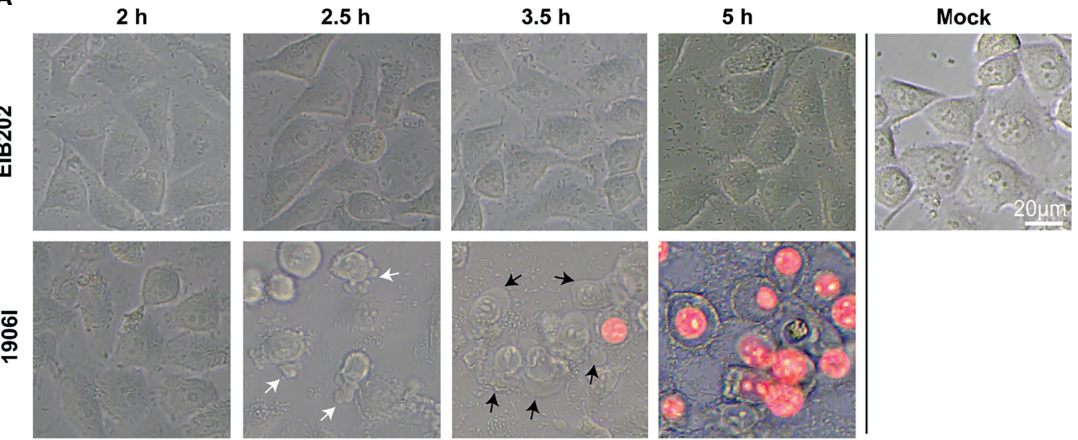

B

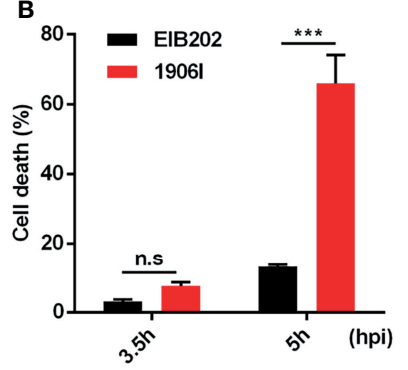

C

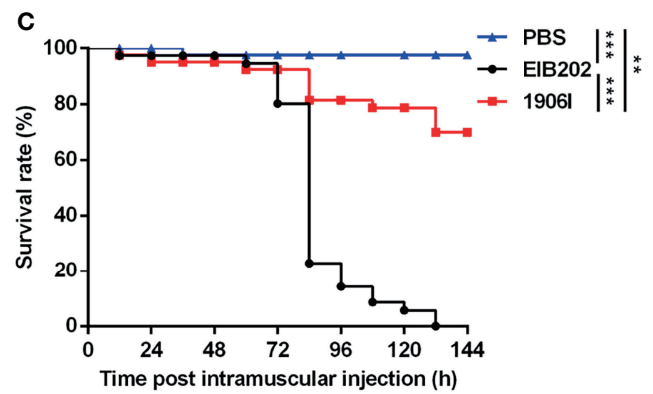

D

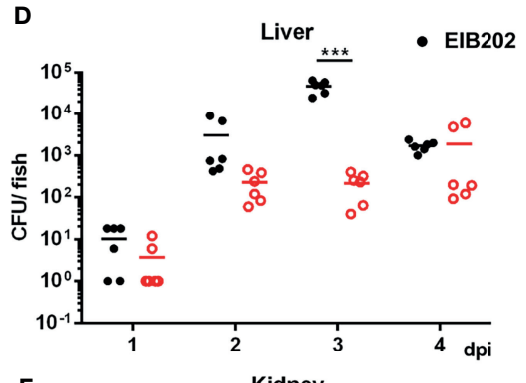

E $\bigcirc 19061$

Spleen

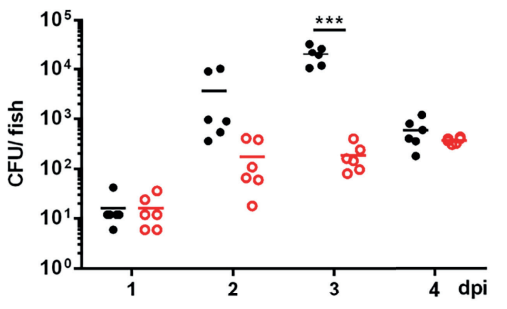

F

Kidney

G

Intestine
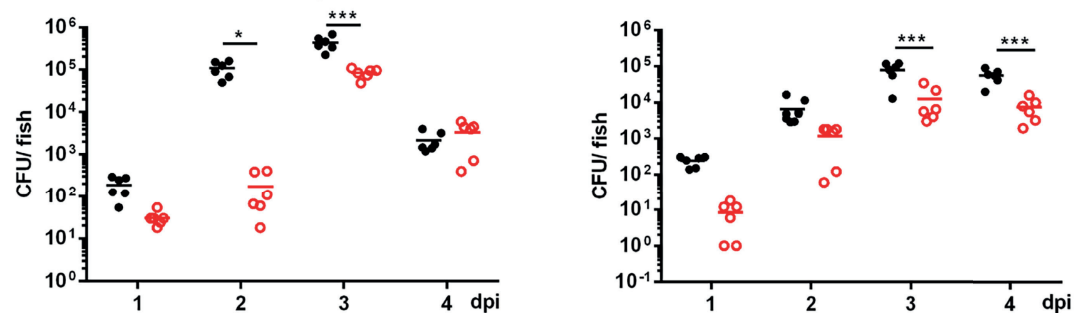

FIGURE 1 | E. piscicida 1906l induced robust membrane-ruptured cell death and showed attenuated bacterial colonization and virulence in zebrafish. (A) Dynamic changes of HeLa cell morphology after infection of wild-type EIB202 or mutant 1906I at an MOI of 25 for the indicated time. White arrows denote the membrane bebbling and black arrows denote membrane swelling and rounding. Propidium iodide (PI) was added to detect the loss of plasma membrane integrity. Scale bar, 20 mm. (B) Lactate dehydrogenase (LDH) release of HeLa cells infected with EIB202 or 1906l for the indicated time. (C-G) Adult zebrafish were intramuscular injected with 50 CFU indicated E. piscicida strains per fish. (C) Survival of zebrafish was monitored for 6 days. PBS as control. ( $n=35$ ). (D-G) Bacteria colonization in liver (D), spleen (E), kidney (F) and intestine (G) of the indicated time points post infection. Five surviving adult zebrafish were pooled as a sample and dissected at the indicated time point post infection, and livers, spleens, kidneys, and intestines were collected and grinded to homogenates for CFU counts, respectively. Results are representative of at least three independent experiments. ${ }^{\star} P<0.05,{ }^{\star \star} P<0.01,{ }^{\star \star \star} P<0.001$; n.s, not significant. [Gehan-Breslow-Wilcoxon test for panel (C),

Two-way ANOVA for panels (B, D-G)].

biofilm formation as well as the production of EPS, and adhesion to HeLa cells (Figures 2B-E). Furthermore, inactivation of ETAE_1905 eliminated the ability of 1906 I to induce cytotoxicity in HeLa cells (Figures 2F, G), suggesting the overexpression of
ETAE_1905 instead of disruption of ETAE_1906 induced both bacterial phonotypes and cell death.

To further verify the role of DGC and c-di-GMP in the regulation of cell death, we treated HeLa cells with azathioprine 


\section{A}
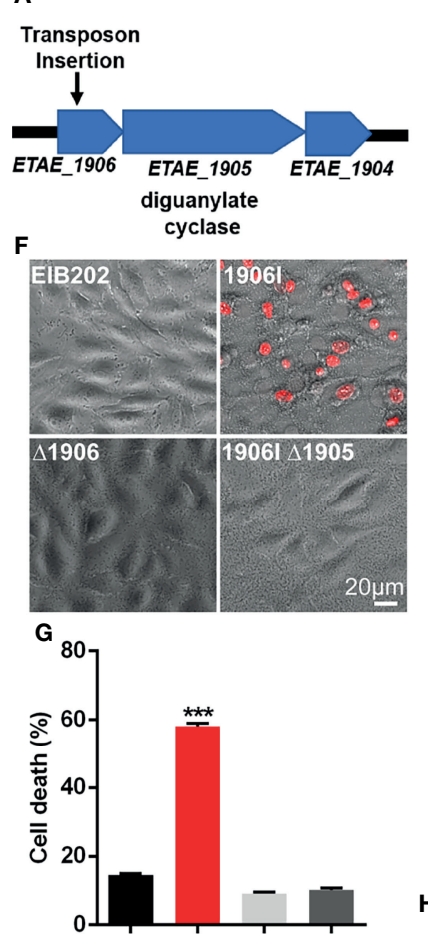
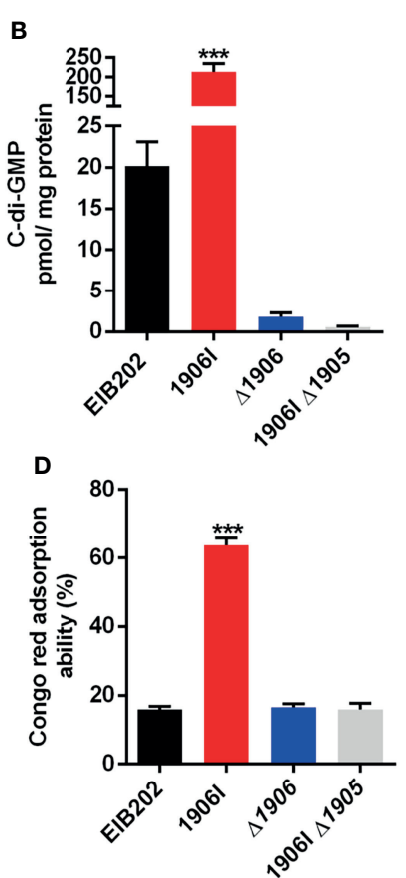

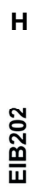

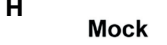

Mock
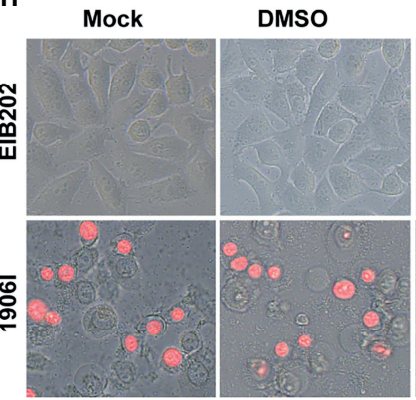
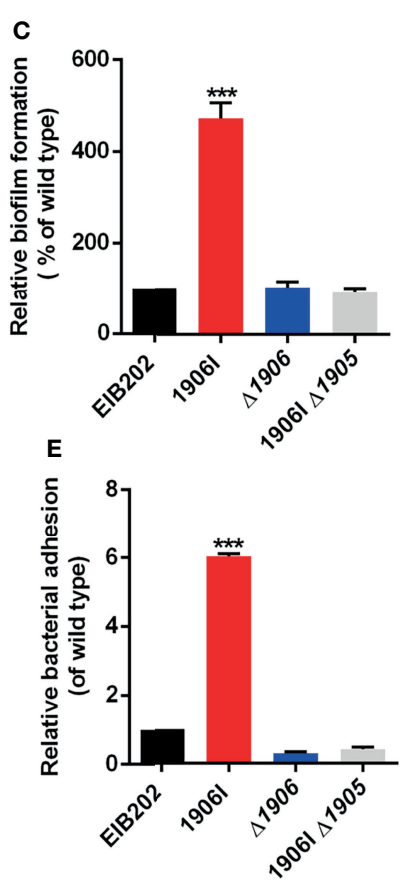

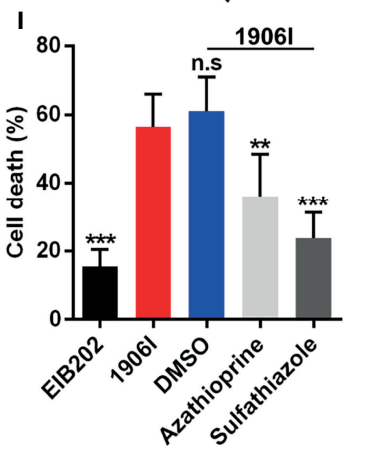

Azathioprine

Sulfathiazole

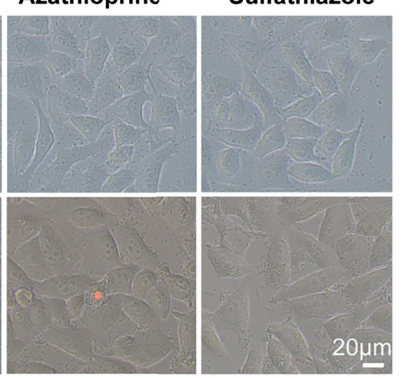

FIGURE 2 | Upregulated c-di-GMP in 1906l strain promoted robust membrane-ruptured cell death. (A) A schematic of transposon insertion location in the genome of 1906 strain. (B) Cytosolic c-di-GMP levels of indicated E. piscicida strains. (C) Biofilm formation of indicated E. piscicida strains detected by crystal violet staining method. (D) Exopolysaccharide production indicated by Congo red adsorption of indicated E. piscicida strains. (E) Bacterial count by agar plating cell lysates from HeLa cells infected by indicated E. piscicida strains (MOI = 100, 1 hpi) after 3 times washing with cold PBS to remove unattached bacteria. (F, G) Cell morphology with PI staining $\mathbf{( F )}$ and LDH release $\mathbf{( G )}$ of Hela cells after infection with indicated E. piscicida strains (MOl=25, 5 hpi). Scale bar, $20 \mu \mathrm{m}$. (H, I) Cell morphology with PI staining $\mathbf{( H )}$ and LDH release $\mathbf{( I )}$ of HeLa cells infected with E. piscicida EIB202 or 1906I (MOI=25, 5 hpi) in the presence of c-di-GMP synthesis inhibitor azathioprine $(50 \mu \mathrm{g} / \mathrm{ml})$, sulfathiazole $(100 \mu \mathrm{g} / \mathrm{ml})$, DMSO. Scale bar, $20 \mu \mathrm{m}$. Results are representative of at least three independent experiments, and error bars denote SD of triplicate wells. ${ }^{\star \star} P<0.01,{ }^{\star \star \star} P<0.001$; n.s, not significant. (One-way ANOVA).

or sulfathiazole, two small molecules that inhibit biosynthesis of c-di-GMP (Antoniani et al., 2010; Antoniani et al., 2013). Cell death and morphological changes induced by the 1906I mutant were inhibited by treatment with either azathioprine or sulfathiazole (Figures 2H, I). Collectively, these results indicate that the 1906I mutant overexpresses the gene encoding DGC leading to increased levels of c-di-GMP which is important for induction of lytic cell death in host cells.

\section{Dysregulated c-di-GMP-Induced Cell Death Is Not Mediated by Caspases or the RIP1/3-MLKL Axis}

Because 1906I-induced cell death showed both apoptotic and necrotic-like features, including membrane blebbing, swelling and membrane rupture, we assessed whether caspase-mediated apoptotic, pyroptotic cell death or necroptosis may be involved in the response to infection with 1906I. To identify the possible 
signaling pathway of host cell death induced by dysregulated cdi-GMP in E. piscicida, we firstly assessed the role of caspases by measuring the ability of cell lysates from HeLa cells to cleave a panel of fluorogenic and chromogenic caspase peptide substrates. As shown in Figure 3A, cell lysates from 1906I-infected HeLa cells did not show difference in their ability to cleave any of the caspase substrates tested when compared with those from EIB202-infected cells (Figure 3A). To further verify this, $\mathrm{z}^{-}$ YVAD-fmk, z-VDVAD-fmk, z-DEVD-fmk, z-WEHD-fmk, zVEID-fmk, z-IETD-fmk, z-LEHD-fmk, and z-VAD-fmk were used to inhibit the activity of caspase $1 / 4$, caspase 2 , caspase $3 / 7$, caspase 5, caspase 6, caspase 8, caspase 9 and all caspases, respectively. Treatment with these panel of caspase inhibitors did not reduce cytotoxicity (Figure 3B) or morphological changes (Figure S3A) in 1906I-infected HeLa cells. These data suggest that 1906I-induced cell death is independent of caspases.

Several types of cell death mediated by caspases culminate in the formation of a lytic pore induced by the cleavage of poreforming proteins Gasdermin D (GSDMD) and Gasdermin E (GSDME) (Shi et al., 2015; Rogers et al., 2017; Wang et al., 2017). To assess a role for GSDMD in cell death induced by the 1906I mutant, we infected wild-type and GSDMD-deficient HeLa cells with 1906I. However, GSDMD deficiency did not prevent the death of HeLa cells induced by 1906I infection (Figure 3C). Moreover, GSDME was not cleaved into the active N-terminal fragment either in HeLa cells or GSDME-highly expressing NCIH226 cells upon E. piscicida strains infection while it was cleaved after treatment with the caspase 3 activator staurosporine (Figure 3D). We also assessed whether 1906I-induced cell death was induced via RIP1/RIP3-mediated necroptosis. We found that cytotoxicity and morphology induced by 1906I were not inhibited by the RIP1 inhibitor necrostatin-1, the RIP3 inhibitor GSK'872, or the MLKL oligomerization inhibitor necrosulfonamide (Figures 3E, F). In accord with these results, MLKL deficiency did not impair the cell death induced by 1906I (Figure 3G). Taken together, these data suggest that the upregulation of c-di-GMP in E. piscicida promotes non-pyroptotic and non-necroptotic membrane-ruptured cell death independent of caspases, Gasdermin D/E, RIP1/3, or MLKL.

\section{C-di-GMP-Mediated Cell Death Is Iron-Dependent}

Because lytic cell death induced by 1906I is neither pyroptosis nor necroptosis, we investigated whether the induced cell death was ferroptosis. To assess this possibility, we first examined whether there is intracellular iron accumulation in response to 1906 infection. The fluorescent probes CA-AM and PGSK, were used to quantitively detect cytosolic labile iron by measuring the quenched fluorescence intensity (Epsztejn et al., 1997; Petrat et al., 1999). Notably, there was an increase in cytosolic labile iron in HeLa cell infected with 1906I or incubation with FeAC that was used as a positive control when compared with EIB202 infection (Figures 4A, B). The increase in labile iron induced by 1906 infection was effectively inhibited by treatment with DFM, an iron chelator (Figures 4A, B). Because mitochondria are main destination and site of iron usage in cellular metabolism ( $\mathrm{Lv}$ and
Shang, 2018), we also tested the mitochondrial labile iron content after infection with 1906I. We found a marked increase in labile iron in the mitochondria of HeLa cells upon 1906I infection (Figure 4C). These results suggest that upregulation of c-diGMP in E. piscicida promotes intracellular labile iron accumulation in host cells. Importantly, treatment with the iron chelator DFM suppressed the cell death induced by 1906I (Figure 4D). Taken together, these results suggest that c-di-GMP of E. piscicida induces cellular iron accumulation and triggers iron-dependent cell death after infection.

\section{Iron Accumulation Induces Mitochondrial Dysfunction and Oxidative Stress}

Labile iron has high chemical redox reactivity which could catalyze excess Fenton reactions leading to the production of ROS and cytotoxicity (Winterbourn, 1995). Because mitochondria are major sites of oxygen consumption and electron transport, redox active labile iron in mitochondria could lead to oxidative stress and cell damage. To assess mitochondrial dysfunction, we labeled the cells with the mitochondrial membrane potential-sensitive dye JC-1 upon $E$. piscicida infection. We found a decrease of red fluorescence and an increase of green fluorescence as $\Delta \Psi \mathrm{m}$ loss in HeLa cells infected with 1906I while the fluorescence in EIB202-infected cells remained unchanged and comparable to that observed in untreated cells (Figure 5A). The loss of mitochondrial potential induced by 1906I infection was inhibited by treatment with DFM (Figure 5A).

Because mitochondrial electron transport chain utilizes a series of electron transfer reactions to generate cellular ATP through oxidative phosphorylation (Nolfi-Donegan et al., 2020), we examined the effect of E. piscicida infection on ATP generation. Infection of HeLa cells with the 1906I mutant induced a reduction of ATP compared with infection with the wild-type EIB202 which was blocked by treatment with the iron chelator DFM (Figure 5B). We also investigated whether iron accumulation caused by 1906I also leads to intracellular oxidative stress. Compared with that observed after infection with EIB202, 1906I induced an increase of mitochondrial superoxide and intracellular ROS generation in HeLa cells, which was inhibited by addition of DFM (Figures 5C, D). Collectively, these results suggest that iron accumulation induces mitochondrial dysfunction and enhances cellular oxidative stress causing cell damage and cytotoxicity.

\section{C-di-GMP-Dysregulation Induces Non-Canonical Ferroptosis Independent of Lipid Peroxidation}

Classical ferroptosis is an iron-dependent oxidative programed cell death characterized by the accumulation of lipid peroxides. Because of the iron-dependent and oxidative features of ferroptosis induced by $1906 \mathrm{I}$ infection, we next assessed whether 1906I triggers ferroptosis through lipid peroxidation. To assess this question, we utilized the lipid peroxidation sensor BODIPY-C11 whose fluorescence shifts from red to green upon oxidation in HeLa cells infected with E. piscicida strains. A 

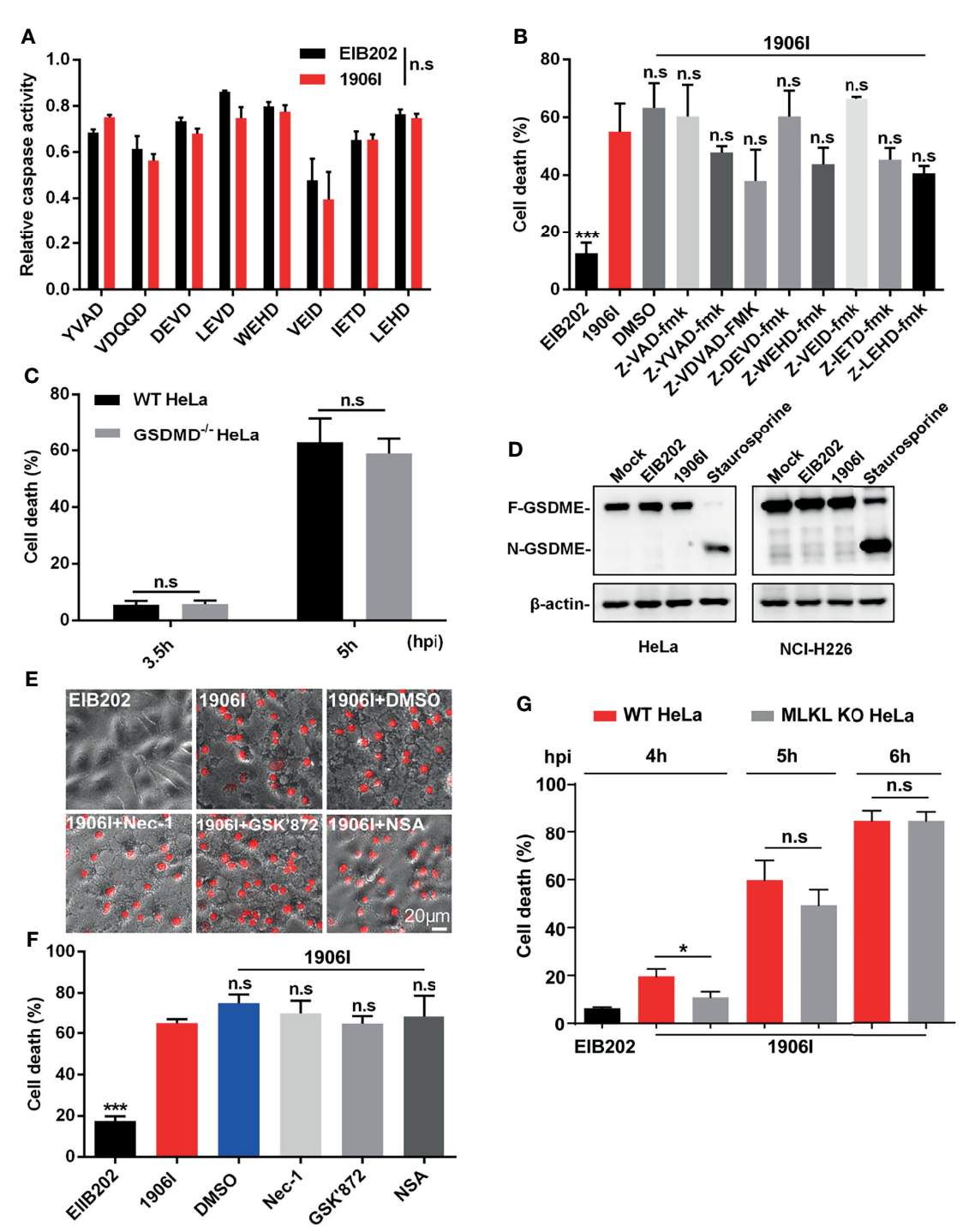

FIGURE 3 | Cell death induced by c-di-GMP-upregulated E. piscicida was independent of caspases, Gasdermin D/E or RIP1/3-MLKL axis. (A) HeLa cells were infected with E. piscicida EIB202 or $1906 \mathrm{I}(\mathrm{MOI}=25,3.5 \mathrm{hpi})$. The relative caspase activity was then measured by incubating cell lysate of infected or uninfected cells with chromogenic substrates of caspase-1 (Ac-YVAD-pNA), caspase-2 (Ac-VDQQD-pNA), caspase-3/7 (Ac-DEVD-pNA), caspase-4 (Ac-LEVD-pNA), caspase-6 (AcVEID-AFC), caspase-8 (Ac-IETD-pNA) and caspase-9 (Ac-LEHD-pNA), or fluorogenic substrates of caspase-5 (Ac-WEHD-AFC). (B) HeLa cells were infected with E.

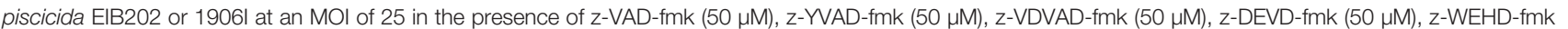
$(20 \mu \mathrm{M}), z$-VEID-fmk $(50 \mu \mathrm{M}), z$-IETD-fmk $(50 \mu \mathrm{M})$, z-LEHD-fmk $(50 \mu \mathrm{M})$, or DMSO. LDH release was detected at $5 \mathrm{~h}$ post infection. (C) LDH release of wild-type or gsdmd $^{-1 /}$ HeLa cells infected with $1906 \mathrm{l}$ strain (MOI=25, 5 hpi). (D) Immunoblots for GSDME cleavage in HeLa or NCl-H226 cells infected with E. piscicida ElB202 or 1906l. HeLa cells treated with staurosporine $(20 \mu \mathrm{M})$ as a positive control, cells without any treatment as a negative control. (E, F) Cell morphology with PI staining

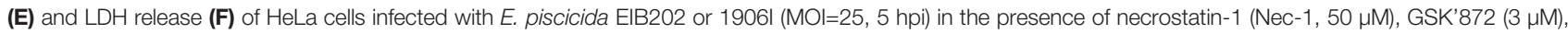
necrosulfonamide (NSA, $20 \mu \mathrm{M}$ ), or DMSO. (G) LDH release of wild-type or m/k ${ }^{-1}$ HeLa cells infected with E. piscicida EIB202 or $1906 \mathrm{l}$ at an MOI of 25 for the indicated time. Scale bar, $20 \mu \mathrm{m}$. Results are representative of at least three independent experiments, and error bars denote SD of triplicate wells. ${ }^{*} P<0.05$ ${ }^{\star \star \star} P<0.001 ;$ n.s, not significant. [Two-way ANOVA for panels (A, C, G) One-way ANOVA for panels (B, F)].

ferroptosis inducer RSL3 induced ferroptosis in HeLa cells (Figure 6E) and an increase of green fluorescence was detected in cells stained with BODIPY-C11 (Figure 6A). In contrast, the fluorescence profile of cells infected by either 1906I or EIB202 was comparable to that observed in untreated cells (Figure 6A). These results suggest that $E$. piscicida infection with wild-type and mutant strains does not induce detectable lipid peroxidation under conditions in which the 1906I mutant induces irondependent cell death.

To further confirm these results, we treated HeLa cells with inhibitors of LOXs, which catalyze the deoxygenation of free and esterified polyunsaturated fatty acids to generate various lipid hydroperoxides (Hassannia et al., 2019). Treatment with PD146176, zileuton, and ML351 effectively inhibited the 

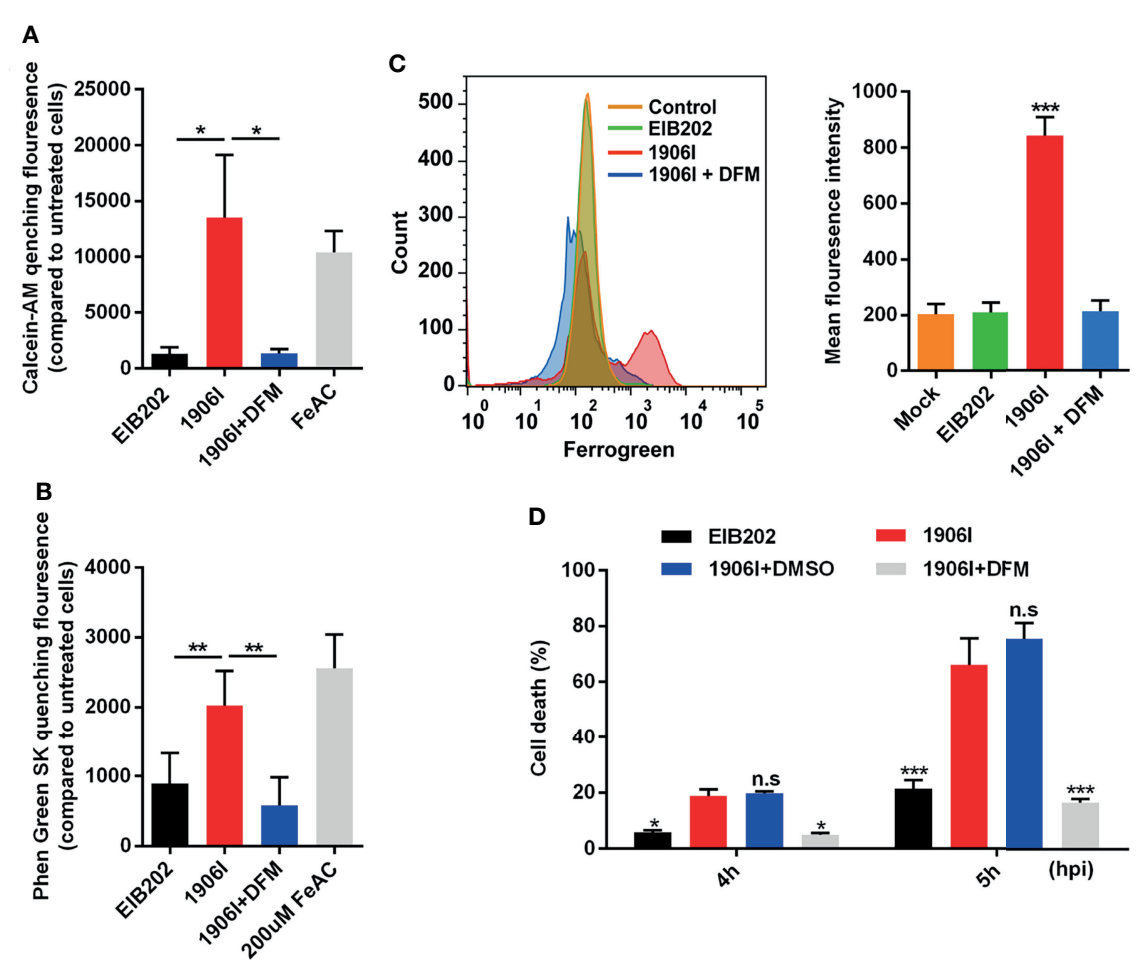

FIGURE 4 | C-di-GMP-upregulated E. piscicida promoted cytosolic and mitochondrial labile iron accumulation triggering an iron-dependent cell death.

(A, B) Calcein-AM (A) and Phen Green SK (B) quenching fluorescence intensity of HeLa cells treated with indicated E. piscicida strains or $200 \mu \mathrm{M}$ ferric ammonium citrate (FeAC) with or without deferoxamine mesylate (DFM, $50 \mu \mathrm{M}$ ) for $3 \mathrm{~h}$ when compared to cells incubated with Opit-MEM only. Fluorescence intensity of cells was measured with a Multiscan Spectrum microplate reader and quenched fluorescence intensity compared to untreated cells reflected the cytosolic labile iron content. (C) Flow cytometric detection of mitochondrial labile iron in HeLa cells infected with E. piscicida EIB202 or $1906 \mathrm{I}$ with or without DFM (50 $\mu$ M) by mitochondria-specific fluorescent probe Mito-FerroGreen. (D) LDH release of HeLa cells infected with E. piscicida EIB202 or 19061 at an MOI of 25 for the indicated time in the presence of DFM $(50 \mu \mathrm{M})$ or DMSO. Results are representative of at least three independent experiments, and error bars denote SD of triplicate wells. ${ }^{\star} P<0.05,{ }^{\star \star} P<0.01,{ }^{\star \star \star} P<0.001 ;$ n.s, not significant. [One-way ANOVA for panels (A-C), Two-way ANOVA for panel (D)].

morphological change and cell death triggered by RSL3 (Figure 6E), but failed to suppress cytotoxicity caused by $1906 \mathrm{I}$ infection (Figure 6B). Because lipid peroxides can be generated not only by LOXs-catalyzed lipid peroxidation, but also by nonenzymatic or auto-oxidation of lipids (Hassannia et al., 2019), we treated the cells with lipophilic antioxidants Fer-1 and Lipro-1 to assess their effects on cytotoxicity caused by 1906 I. Fer-1 and Lipro-1 suppressed RSL3-induced ferroptosis (Figure 6E), but they were unable to inhibit morphological changes or cytotoxicity in 1906I-infected HeLa cells (Figures 6C, D). Collectively, these results suggest that dysregulated c-di-GMP in E. piscicida promotes cell damage and death through a non-canonical ferroptosis pathway that is independent of lipid peroxidation.

\section{DISCUSSION}

C-di-GMP is a universal bacterial secondary messenger that is synthesized from two GTP molecules by DGC and degraded by phosphodiesterase (Romling et al., 2013). The cytosolic c-di-GMP content is tightly controlled by DGCs and phosphodiesterases and regulates bacterial function by binding to downstream effectors in response to environmental cues (Romling et al., 2013; Hall and Lee, 2018). High levels of c-di-GMP facilitate the synthesis of adhesins and biofilm matrix components including EPS and interfered with motility and virulence functions while decreased c-di-GMP is associated with opposite phenotypes (Hengge, 2009). As an important secondary messenger widely distributed in diverse bacteria, increasing evidence suggest that c-di-GMP plays a role in bacterial pathogenesis. For example, low intracellular c-di-GMP levels promotes expression of virulence factors in Vibrio cholerae while elevated intracellular c-di-GMP attenuates virulence in a neonatal mouse model of cholera infection (Tischler and Camilli, 2005). Likewise, deletion of $b p d A$ and $b p d B$, which exhibited the cyclic-di-GMP phosphodiesterases phenotype in Brucella melitensis reduced its virulence in mice while deletion of $C g s B$ displaying c-di-GMP synthase phenotype increased virulence (Petersen et al., 2011). High c-di-GMP levels also suppress acute infections of Yersinia pestis and Borrelia burgdorferi by upregulating the production of extracellular biofilm matrix (Bobrov et al., 2011; Sultan et al., 2011). Here, we revealed that transposon insertion in E. piscicida 1906 I resulted in activation of DGC, causing an increase of cytosolic c-di-GMP, and enhanced production of EPS, biofilm 
A
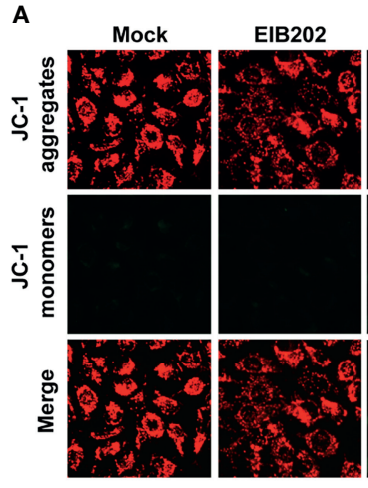

C

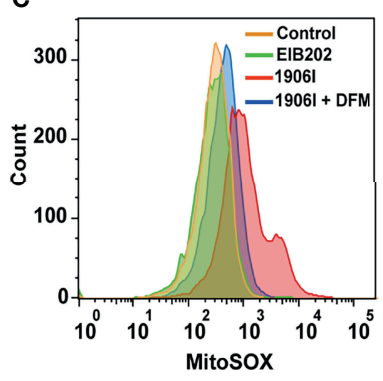

1906 I
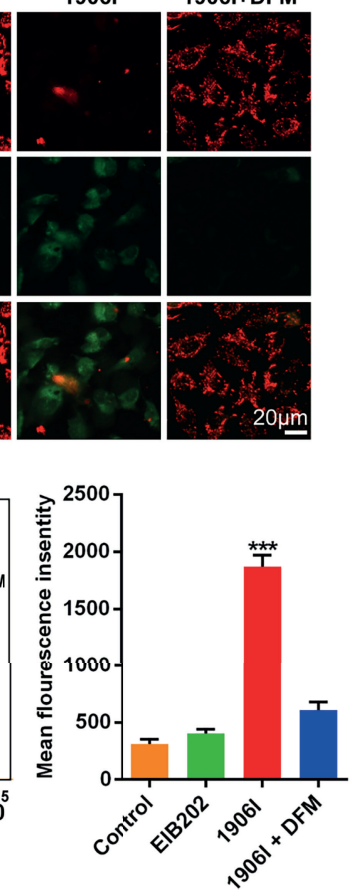

1906I+DFM

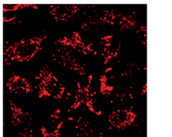

.

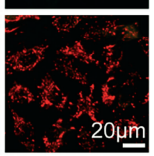

B

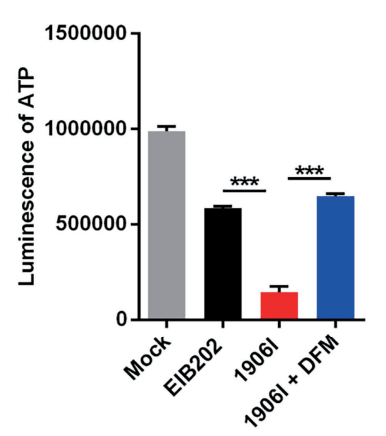

D

ROS (DCFH-DA)

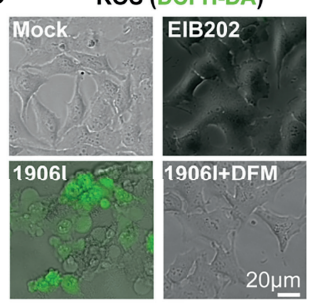

FIGURE 5 | Iron accumulation induced decrease of mitochondrial potential and ATP production and enhanced mitochondrial and cytosolic ROS generation. HeLa cells were infected with indicated $E$. piscicida strains at an $\mathrm{MOI}$ of 25 with or without DFM $(50 \mu \mathrm{M})$. (A) Mitochondrial membrane potential ( $\Delta \Psi \mathrm{\Psi m})$ of HeLa cells was detected by JC-1 staining and observed by fluorescent microscopy at $3.5 \mathrm{~h}$ post bacterial infection. The double staining of cells by JC- 1 is visible either as green for JC-1 monomers (low $\Delta \Psi \mathrm{m}$ ) or red for JC-1 aggregates (high $\Delta \Psi$ ). Scale bar, $20 \mu \mathrm{m}$. (B) ATP level of HeLa cells was measured by a luminescent ATP kit at $3.5 \mathrm{~h}$ post bacterial infection. (C) Flow cytometric detection of mitochondrial ROS by MitoSOX at $3.5 \mathrm{~h}$ post bacterial infection. (D) Determination of cellular ROS by DCFHDA probe. Fluorescent microscopic images were taken at $3.5 \mathrm{~h}$ post bacterial infection. Scale bar, $20 \mu \mathrm{m}$. Results are representative of at least three independent experiments, and error bars denote SD of triplicate wells. ${ }^{\star \star \star} P<0.001$ (One-way ANOVA).

formation, and bacterial adhesion as well as increased cytotoxicity in eukaryotic cells which blocked by inhibitors of c-di-GMP synthesis. We found that elevated c-di-GMP attenuated $E$. piscicida's virulence in zebrafish which was correlated with reduced bacterial colonization in several organs early in infection. These results provide support for a contribution of cdi-GMP signaling to bacterial pathogenesis and in particular $E$. piscicida infection. However, further investigations are needed to figure out the specific mechanism of c-di-GMP signaling in regulating bacterial infection including what and how specific cidi-GMP-regulated component affects eukaryotic cellular events since it remains to be possible that the DGC overexpression in 1906I might cause these phenotypes through a non-c-diGMP pathway.

Ferroptosis has been reported to be catalyzed and initiated by iron through accelerating Fenton reaction to generate ROS, and executed by excessive peroxidation of polyunsaturated fatty acids in cell membranes (Dixon et al., 2012; Hassannia et al., 2019). Ferroptosis-inducers RSL3 and Erastin can directly or indirectly affect glutathione peroxidase 4 resulting in a decrease of antioxidant capacity and accumulation of lipid ROS ultimately leading to cell death (Dixon et al., 2012; Li et al., 2020). Accordingly, ferroptosis can be blocked by iron chelators or lipid peroxides-related inhibitors including LOXs inhibitors and lipophilic antioxidants (Dixon et al., 2012; Yang et al., 2014; Hassannia et al., 2019; Li et al., 2020). Morphologically, cells undergoing ferroptosis have a typical necrotic morphology along with small aberration and decreased potential of mitochondria, but no clear hallmarks of apoptosis (Li et al., 2020). An important finding of our work is the link of dysregulation of c-di-GMP to lytic cell death in host cells infected with E. piscicida. We found that increased production of c-di-GMP by E. piscicida triggers ferroptosis (Figure 7). However, unlike classical ferroptosis or other regulated necrotic cell death, morphologically, cells infected with E. piscicida $1906 \mathrm{I}$ also exhibited apoptotic features including membrane blebbing and formation of balloon-like protrusions. In addition, unlike classical ferroptosis, we found no evidence for a role of lipid peroxidation in ferroptosis induced by $1906 \mathrm{I}$ infection. The results were further validated by using diverse LOXs inhibitors and lipophilic antioxidants, all of which failed to inhibit morphological changes and cell death in response to infection with E. piscicida 1906I. Thus, our studies suggest that cell death induced by the $1906 \mathrm{I}$ mutant represents a non-canonical ferroptosis pathway that is independent of lipid peroxidation. Although the iron-dependent mediators that are responsible for non-canonical ferroptosis in our system remain unclear, it is possible that non-lipid toxic molecules or different types of lipids could induce oxidative stress and cell damage. Additional 


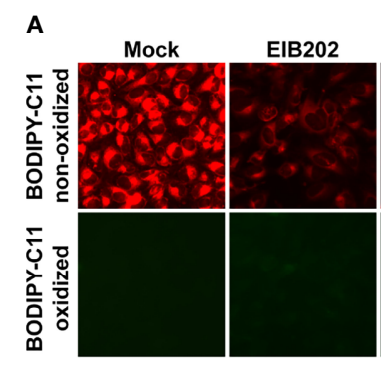

C

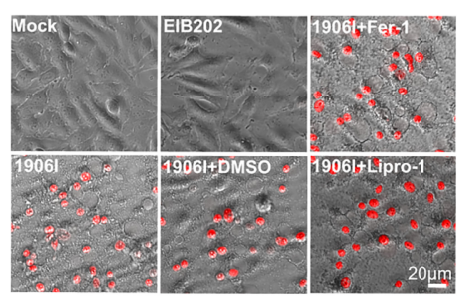

D

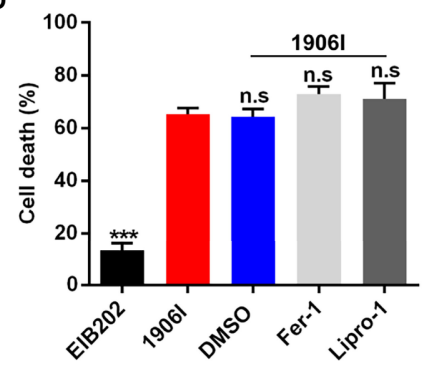

B

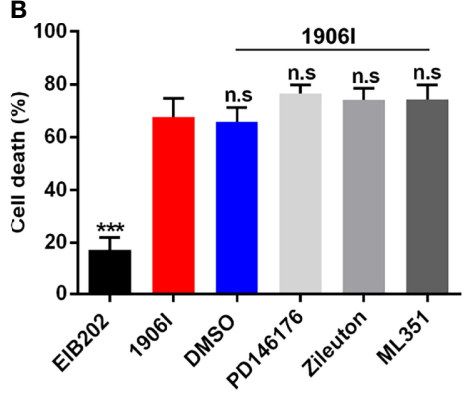

E
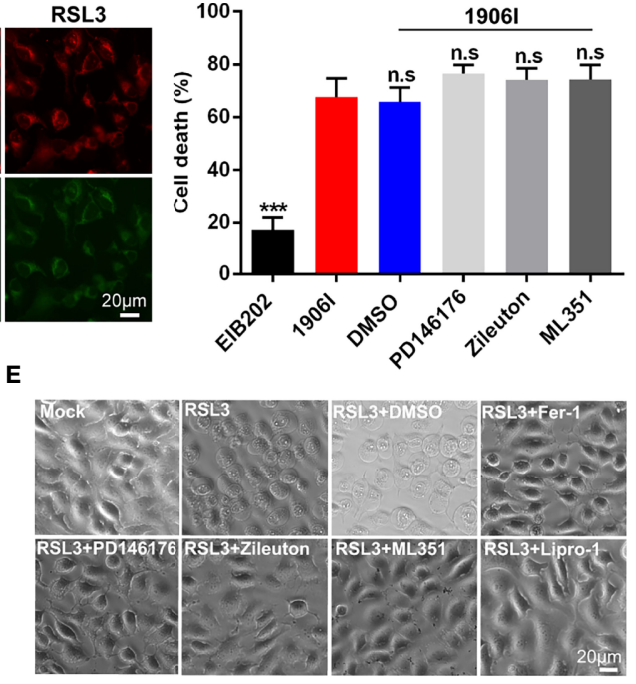

FIGURE 6 | Cell death induced by c-di-GMP-upregulated E. piscicida was independent of lipid peroxidation. (A) Detection of lipid peroxidation by BODIPY-C11 in HeLa cells treated with indicated E. piscicida strains (MOI=25) or RSL3. Fluorescent microscopic images were taken at $3.5 \mathrm{~h}$ post bacterial infection or RSL3 treatment. Untreated cells as a negative control and RSL3-treated cells as a positive control. Scale bar, $20 \mu \mathrm{m}$. (B) LDH release of HeLa cells infected with E.

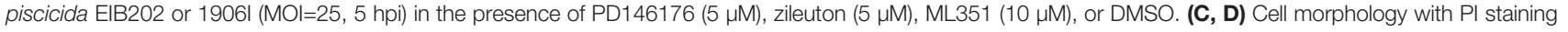
(C) and LDH release (D) of HeLa cells infected with E. piscicida EIB202 or 1906I (MOI=25, 5 hpi) in the presence of ferrostatin-1 (Fer-1, 20 MM), liproxstatin-1 (Lipro1, $20 \mu \mathrm{M})$, or DMSO. Scale bar for the images, $20 \mu \mathrm{m}$. (E) Cell morphology of RSL3-treated HeLa cells with PD146176 (5 $\mu \mathrm{M})$, zileuton (5 $\mu \mathrm{M})$, ML351 (10 $\mu \mathrm{M})$, Fer$1(20 \mu \mathrm{M})$, lipro-1 $(20 \mu \mathrm{M})$, or DMSO. Scale bar, $20 \mu \mathrm{m}$. Results are representative of at least three independent experiments, and error bars denote SD of triplicate wells. ${ }^{\star \star *} P<0.001 ;$ n.s, not significant. (One-way ANOVA).

work is needed to understand how dysregulated c-di-GMP induces iron accumulation and the iron-dependent molecules that mediate lytic cell death.

Previous studies of ferroptosis mostly focused on its role in sterile disease such as neurodegeneration, acute kidney injury and cancer, but few studies have addressed the relevance of ferroptosis during pathogen infection. A recent study found that Pseudomonas aeruginosa can utilize lipoxygenase (pLoxA) to oxidize host arachidonic acid-phosphatidylethanolamines (AA-PE) to 15hydroperoxy-AA-PE triggering ferroptosis in human bronchial epithelial cells (Dar et al., 2018). Mycobacterium tuberculosis was also reported to induce accumulation of labile iron and lipid peroxidation in macrophages both in vitro and in vivo (Amaral et al., 2019). In the present work, we found that upregulation of c-diGMP in E. piscicida strain can also lead to increased labile iron, mitochondrial superoxide inducing cell death which can be blocked by iron chelator in HeLa cells. However, unlike Pseudomonas. aeruginosa or Mycobacterium tuberculosis, E. piscicida induced a non-canonical ferroptosis which is independent of lipid ROS and cannot be suppressed by Fer-1 or other lipophilic antioxidants. Moreover, animals infected with Mycobacterium tuberculosis display prominent decrease in bacterial loads when ferroptosis is suppressed (Amaral et al., 2019). In contrast, E. piscicida-induced ferroptosis correlated with protective function in the host during infection in that zebrafish infected with the mutant E. piscicida 1906I showed reduced pathogen loads and increased survival. These differences could be explained by the use of a different model of pathogen and host infection, differences in the ferroptosis pathway or the fact that in our model ferroptosis was trigger by c-di-GMP dysregulation in the mutant $1906 \mathrm{I}$.

Collectively, our study shows that elevated c-di-GMP in E. piscicida triggers an iron-dependent, non-canonical ferroptosis, which is independent of lipid peroxidation, and reduces pathogen virulence in zebrafish (Figure 7), indicating the 


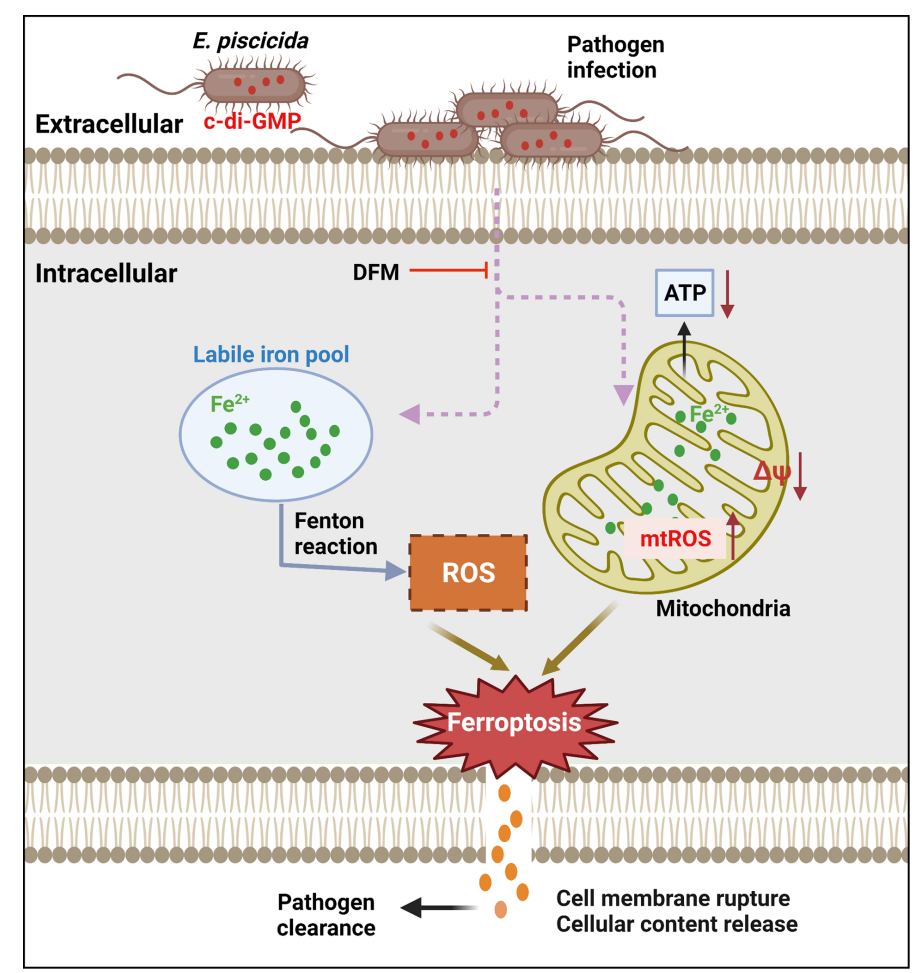

FIGURE 7 | Summary of proposed mechanism of bacterial c-di-GMP promoting non-canonical ferroptosis. Elevated c-di-GMP in E. piscicida promotes cellular labile iron accumulation resulting in decrease of mitochondrial potential and ATP production, and increase of mitochondrial and cytosolic ROS generation triggering a noncanonical ferroptosis which is independent of lipid peroxidation and could be inhibited by iron chelator DFM. The dysregulation of c-di-GMP in E. piscicida also attenuates its colonization and virulence in vivo.

importance of tight regulation of intracellular c-di-GMP during infection and presenting a non-canonical ferroptotic pathway independent of lipid peroxidation that can be triggered by pathogen infection.

\section{DATA AVAILABILITY STATEMENT}

The original contributions presented in the study are included in the article/Supplementary Material. Further inquiries can be directed to the corresponding author.

\section{ETHICS STATEMENT}

The animal study was reviewed and approved by Institutional Animal Care and Use Committee of East China University of Science and Technology (No. 2006272).

\section{AUTHOR CONTRIBUTIONS}

QL conceived and supervised the study. YWen conducted most of the experiments with the help of YWang and SC. YWen and DY wrote the manuscript. GN, XZ, and YZ critically revised the manuscript. All authors discussed the results and commented on the manuscript. All authors contributed to the article and approved the submitted version.

\section{FUNDING}

This work was supported by Frontier Science Research Base of Optogenetic Techniques for Cell Metabolism grant 2021 Sci \& Tech 03-28 (Shanghai Municipal Education Commission), National Natural Science Funds for Distinguished Young Scholar (No. 32025038), and China Postdoctoral Science Foundation (No. 2020M671033). YWen was supported by the State Scholarship Fund from China Scholarship Council (No. 201906740067).

\section{ACKNOWLEDGMENTS}

We would like to thank Dr. Feng Shao from NIBs for providing gsdmd-deficient HeLa cells.

\section{SUPPLEMENTARY MATERIAL}

The Supplementary Material for this article can be found online at: https://www.frontiersin.org/articles/10.3389/fcimb.2022. 825824/full\#supplementary-material 


\section{REFERENCES}

Amaral, E. P., Costa, D. L., Namasivayam, S., Riteau, N., Kamenyeva, O., Mittereder, L., et al. (2019). A Major Role for Ferroptosis in Mycobacterium Tuberculosis-Induced Cell Death and Tissue Necrosis. J. Exp. Med. 216, 556570. doi: 10.1084/jem.20181776

Antoniani, D., Bocci, P., Maciag, A., Raffaelli, N., and Landini, P. (2010). Monitoring of Diguanylate Cyclase Activity and of Cyclic-Di-GMP Biosynthesis by Whole-Cell Assays Suitable for High-Throughput Screening of Biofilm Inhibitors. Appl. Microbiol. Biotechnol. 85, 1095-1104. doi: 10.1007/ s00253-009-2199-x

Antoniani, D., Rossi, E., Rinaldo, S., Bocci, P., Lolicato, M., Paiardini, A., et al. (2013). The Immunosuppressive Drug Azathioprine Inhibits Biosynthesis of the Bacterial Signal Molecule Cyclic-Di-GMP by Interfering With Intracellular Nucleotide Pool Availability. Appl. Microbiol. Biotechnol. 97, 7325-7336. doi: $10.1007 / \mathrm{s} 00253-013-4875-0$

Ashida, H., Mimuro, H., Ogawa, M., Kobayashi, T., Sanada, T., Kim, M., et al. (2011). Cell Death and Infection: A Double-Edged Sword for Host and Pathogen Survival. J. Cell Biol. 195, 931-942. doi: 10.1083/jcb.201108081

Bobrov, A. G., Kirillina, O., Ryjenkov, D. A., Waters, C. M., Price, P. A., Fetherston, J. D., et al. (2011). Systematic Analysis of Cyclic Di-GMP Signalling Enzymes and Their Role in Biofilm Formation and Virulence in Yersinia Pestis. Mol. Microbiol. 79, 533-551. doi: 10.1111/j.13652958.2010.07470.x

Cervantes, J., Nagata, T., Uchijima, M., Shibata, K., and Koide, Y. (2008). Intracytosolic Listeria Monocytogenes Induces Cell Death Through Caspase1 Activation in Murine Macrophages. Cell. Microbiol. 10, 41-52. doi: 10.1111/ j.1462-5822.2007.01012.x

Chen, H., Yang, D., Han, F., Tan, J., Zhang, L., Xiao, J., et al. (2017). The Bacterial T6SS Effector EvpP Prevents NLRP3 Inflammasome Activation by Inhibiting the $\mathrm{Ca}(2+)$-Dependent MAPK-Jnk Pathway. Cell Host Microbe 21, 47-58. doi: 10.1016/j.chom.2016.12.004

Chen, S., Yang, D., Wen, Y., Jiang, Z., Zhang, L., Jiang, J., et al. (2018). Dysregulated Hemolysin Liberates Bacterial Outer Membrane Vesicles for Cytosolic Lipopolysaccharide Sensing. PloS Pathog. 14, e1007240. doi: 10.1371/ journal.ppat.1007240

Dar, H. H., Tyurina, Y. Y., Mikulska-Ruminska, K., Shrivastava, I., Ting, H. C., Tyurin, V. A., et al. (2018). Pseudomonas Aeruginosa Utilizes Host Polyunsaturated Phosphatidylethanolamines to Trigger Theft-Ferroptosis in Bronchial Epithelium. J. Clin. Invest. 128, 4639-4653. doi: 10.1172/JC199490

Dewoody, R., Merritt, P. M., Houppert, A. S., and Marketon, M. M. (2011). YopK Regulates the Yersinia Pestis Type III Secretion System From Within Host Cells. Mol. Microbiol. 79, 1445-1461. doi: 10.1111/j.1365-2958.2011.07534.x

Dixon, S. J., Lemberg, K. M., Lamprecht, M. R., Skouta, R., Zaitsev, E. M., Gleason, C. E., et al. (2012). Ferroptosis: An Iron-Dependent Form of Nonapoptotic Cell Death. Cell 149, 1060-1072. doi: 10.1016/j.cell.2012.03.042

Edwards, R. A., Keller, L. H., and Schifferli, D. M. (1998). Improved Allelic Exchange Vectors and Their Use to Analyze 987P Fimbria Gene Expression. Gene 207, 149-157. doi: 10.1016/S0378-1119(97)00619-7

Epsztejn, S., Kakhlon, O., Glickstein, H., Breuer, W., and Cabantchik, I. (1997). Fluorescence Analysis of the Labile Iron Pool of Mammalian Cells. Anal. Biochem. 248, 31-40. doi: 10.1006/abio.1997.2126

Hall, C. L., and Lee, V. T. (2018). Cyclic-Di-GMP Regulation of Virulence in Bacterial Pathogens. WIREs RNA 9, e1454. doi: 10.1002/wrna.1454

Hassannia, B., Vandenabeele, P., and Vanden, B. T. (2019). Targeting Ferroptosis to Iron Out Cancer. Cancer Cell 35, 830-849. doi: 10.1016/j.ccell.2019.04.002

Hengge, R. (2009). Principles of C-Di-GMP Signalling in Bacteria. Nat. Rev. Microbiol. 7, 263-273. doi: 10.1038/nrmicro2109

He, T. T., Zhou, Y., Liu, Y. L., Li, D., Nie, P., Li, A., et al. (2020). Edwardsiella Piscicida Type III Protein EseJ Suppresses Apoptosis Through Down Regulating Type 1 Fimbriae, Which Stimulate the Cleavage of Caspase-8. Ceell. Microbiol. 22, e13193. doi: 10.1111/cmi.13193

Imre, G. (2020). The Involvement of Regulated Cell Death Forms in Modulating the Bacterial and Viral Pathogenesis. Int. Rev. Cell. Mol. Biol. 353, 211-253. doi: 10.1016/bs.ircmb.2019.12.008

Jiang, J., Wang, W., Sun, F., Zhang, Y., Liu, Q., and Yang, D. (2021). Bacterial Infection Reinforces Host Metabolic Flux From Arginine to Spermine for
NLRP3 Inflammasome Evasion. Cell Rep. 34, 108832. doi: 10.1016/ j.celrep.2021.108832

Jorgensen, I., Rayamajhi, M., and Miao, E. A. (2017). Programmed Cell Death as a Defence Against Infection. Nat. Rev. Immunol. 17, 151-164. doi: 10.1038/ nri.2016.147

Katagiri, N., Shobuike, T., Chang, B., Kukita, A., and Miyamoto, H. (2012). The Human Apoptosis Inhibitor NAIP Induces Pyroptosis in Macrophages Infected With Legionella Pneumophila. Microbes Infect. 14, 1123-1132. doi: 10.1016/j.micinf.2012.03.006

Labbe, K., and Saleh, M. (2008). Cell Death in the Host Response to Infection. Cell Death Diffre 15, 1339-1349. doi: 10.1038/cdd.2008.91

Leung, K. Y., Siame, B. A., Tenkink, B. J., Noort, R. J., and Mok, Y. K. (2012). Edwardsiella Tarda - Virulence Mechanisms of an Emerging Gastroenteritis Pathogen. Microbies Infect. 14, 26-34. doi: 10.1016/j.micinf.2011.08.005

Leung, K. Y., Wang, Q., Yang, Z., and Siame, B. (2019). Edwardsiella Piscicida: A Versatile Emerging Pathogen of Fish. Virulence 10, 555-567. doi: 10.1080/ 21505594.2019.1621648

Li, J., Cao, F., Yin, H. L., Huang, Z. J., Lin, Z. T., Mao, N., et al. (2020). Ferroptosis: Past, Present and Future. Cell Death Dis. 11, 88. doi: 10.1038/s41419-020-2298-2

Lv, H., and Shang, P. (2018). The Significance, Trafficking and Determination of Labile Iron in Cytosol, Mitochondria and Lysosomes. Metallomics 10, 899-916. doi: 10.1039/C8MT00048D

Miao, E. A., Leaf, I. A., Treuting, P. M., Mao, D. P., Dors, M., Sarkar, A., et al. (2010). Caspase-1-Induced Pyroptosis is an Innate Immune Effector Mechanism Against Intracellular Bacteria. Nat. Immunol. 11, 1136-1142. doi: $10.1038 /$ ni.1960

Mohanty, B. R., and Sahoo, P. K. (2007). Edwardsiellosis in Fish: A Brief Review. J. Biosci. 32, 1331-1344. doi: 10.1007/s12038-007-0143-8

Nolfi-Donegan, D., Braganza, A., and Shiva, S. (2020). Mitochondrial Electron Transport Chain: Oxidative Phosphorylation, Oxidant Production, and Methods of Measurement. Redox Biol. 37, 101674. doi: 10.1016/ j.redox.2020.101674

Petersen, E., Chaudhuri, P., Gourley, C., Harms, J., and Splitter, G. (2011). Brucella Melitensis Cyclic Di-GMP Phosphodiesterase BpdA Controls Expression of Flagellar Genes. J. Bacteriol. 193, 5683-5691. doi: 10.1128/JB.00428-11

Petrat, F., Rauen, U., and de Groot, H. (1999). Determination of the Chelatable Iron Pool of Isolated Rat Hepatocytes by Digital Fluorescence Microscopy Using the Fluorescent Probe, Phen Green SK. Hepatology 29, 1171-1179. doi: 10.1002/hep.510290435

Rogers, C., Fernandes-Alnemri, T., Mayes, L., Alnemri, D., Cingolani, G., and Alnemri, E. S. (2017). Cleavage of DFNA5 by Caspase-3 During Apoptosis Mediates Progression to Secondary Necrotic/Pyroptotic Cell Death. Nat. Commun. 8, 14128. doi: 10.1038/ncomms14128

Romling, U., Galperin, M. Y., and Gomelsky, M. (2013). Cyclic Di-GMP: The First 25 Years of a Universal Bacterial Second Messenger. Microbiol. Mol. Biol. Rev. 77, 1-52. doi: 10.1128/MMBR.00043-12

Shi, J., Zhao, Y., Wang, K., Shi, X., Wang, Y., and Huang, H. (2015). Cleavage of GSDMD by Inflammatory Caspases Determines Pyroptotic Cell Death. Nature 526, 660-665. doi: 10.1038/nature15514

Sultan, S. Z., Pitzer, J. E., Boquoi, T., Hobbs, G., Miller, M. R., and Motaleb, M. A. (2011). Analysis of the HD-GYP Domain Cyclic Dimeric GMP Phosphodiesterase Reveals a Role in Motility and the Enzootic Life Cycle of Borrelia Burgdorferi. Infect. Immun. 79, 3273-3283. doi: 10.1128/IAI.05153-11

Tischler, A. D., and Camilli, A. (2005). Cyclic Diguanylate Regulates Vibrio Cholerae Virulence Gene Expression. Infect. Immun. 73, 5873-5882. doi: 10.1128/IAI.73.9.5873-5882.2005

Wang, Y., Gao, W., Shi, X., Ding, J., Liu, W., He, H., et al. (2017). Chemotherapy Drugs Induce Pyroptosis Through Caspase-3 Cleavage of a Gasdermin. Nature 547, 99-103. doi: 10.1038/nature22393

Wang, Q., Yang, M., Xiao, J., Wu, H., Wang, X., Lv, Y., et al. (2009). Genome Sequence of the Versatile Fish Pathogen Edwardsiella Tarda Provides Insights Into its Adaptation to Broad Host Ranges and Intracellular Niches. PloS One 4, e7646. doi: 10.1371/journal.pone.0007646

Warren, S. E., Mao, D. P., Rodriguez, A. E., Miao, E. A., and Aderem, A. (2008). Multiple Nod-Like Receptors Activate Caspase 1 During Listeria Monocytogenes Infection. J. Immunol. 180, 7558-7564. doi: 10.4049/ jimmunol.180.11.7558 
Wen, Y., Chen, S., Jiang, Z., Wang, Z., Tan, J., Hu, T., et al. (2019). Dysregulated Haemolysin Promotes Bacterial Outer Membrane Vesicles-Induced PyroptoticLike Cell Death in Zebrafish. Cell. Microbiol. 21, e13010. doi: 10.1111/cmi.13010 Winterbourn, C. C. (1995). Toxicity of Iron and Hydrogen Peroxide: The Fenton Reaction. Toxicol. Lett. 82-83, 969-974. doi: 10.1016/0378-4274(95)03532-X

Xu, W., Gu, Z., Zhang, L., Zhang, Y., Liu, Q., and Yang, D. (2018). Edwardsiella Piscicida Virulence Effector Trxlp Promotes the NLRC4 Inflammasome Activation During Infection. Microb. Pathog. 123, 496-504. doi: 10.1016/j.micpath.2018.08.016

Yang, G., Billings, G., Hubbard, T. P., Park, J., Leung, S., Liu, K. Y., et al. (2017). TimeResolved Transposon Insertion Sequencing Reveals Genome-Wide Fitness Dynamics During Infection. $m B I O$ 8, e01581-e01517. doi: 10.1128/mBio.01581-17

Yang, W. S., SriRamaratnam, R., Welsch, M. E., Shimada, K., Skouta, R., Viswanathan, V. S., et al. (2014). Regulation of Ferroptotic Cancer Cell Death by GPX4. Cell 156, 317-331. doi: 10.1016/j.cell.2013.12.010

Yang, J., Zhao, Y., Shi, J., and Shao, F. (2013). Human NAIP and Mouse NAIP1 Recognize Bacterial Type III Secretion Needle Protein for Inflammasome Activation. Proc. Natl. Acad. Sci. U. S. A. 110, 14408-14413. doi: 10.1073/pnas.1306376110
Conflict of Interest: The authors declare that the research was conducted in the absence of any commercial or financial relationships that could be construed as a potential conflict of interest.

Publisher's Note: All claims expressed in this article are solely those of the authors and do not necessarily represent those of their affiliated organizations, or those of the publisher, the editors and the reviewers. Any product that may be evaluated in this article, or claim that may be made by its manufacturer, is not guaranteed or endorsed by the publisher.

Copyright $\odot 2022$ Wen, Wang, Chen, Zhou, Zhang, Yang, Núñez and Liu. This is an open-access article distributed under the terms of the Creative Commons Attribution License (CC BY). The use, distribution or reproduction in other forums is permitted, provided the original author(s) and the copyright owner(s) are credited and that the original publication in this journal is cited, in accordance with accepted academic practice. No use, distribution or reproduction is permitted which does not comply with these terms. 